\title{
Cosmological constraints from H II starburst galaxy, quasar angular size, and other measurements
}

\author{
Shulei Cao, ${ }^{1 \star}$ Joseph Ryan,${ }^{2} \dagger$ Bharat Ratra ${ }^{1} \ddagger$ \\ ${ }^{1}$ Department of Physics, Kansas State University, 116 Cardwell Hall, Manhattan, KS 66502, USA \\ ${ }^{2}$ Department of Physics, Southern Methodist University, Dallas, TX 75275, USA
}

Accepted XXX. Received YYY; in original form ZZZ

\begin{abstract}
We compare the constraints from two (2019 and 2021) compilations of H II starburst galaxy (H IIG) data and test the model-independence of quasar angular size (QSO) data using six spatially flat and non-flat cosmological models. We find that the new 2021 compilation of H IIG data generally provides tighter constraints and prefers lower values of cosmological parameters than those from the $2019 \mathrm{H}$ IIG data. QSO data by themselves give relatively model-independent constraints on the characteristic linear size, $l_{\mathrm{m}}$, of the QSOs within the sample. We also use Hubble parameter $(H(z))$, baryon acoustic oscillation (BAO), Pantheon Type Ia supernova (SN Ia) apparent magnitude (SN-Pantheon), and DES-3yr binned SN Ia apparent magnitude (SN-DES) measurements to perform joint analyses with HIIG and QSO angular size data, since their constraints are not mutually inconsistent within the six cosmological models we study. A joint analysis of $H(z)$, BAO, SN-Pantheon, SN-DES, QSO, and the newest compilation of $\mathrm{H}$ IIG data provides almost model-independent summary estimates of the Hubble constant, $H_{0}=69.7 \pm 1.2 \mathrm{~km} \mathrm{~s}^{-1} \mathrm{Mpc}^{-1}$, the non-relativistic matter density parameter, $\Omega_{\mathrm{m}_{0}}=0.293 \pm 0.021$, and $l_{\mathrm{m}}=10.93 \pm 0.25 \mathrm{pc}$.
\end{abstract}

Key words: cosmological parameters - dark energy - cosmology: observations

\section{INTRODUCTION}

Many observations indicate that the Universe is currently in a phase of accelerated expansion, however, the theory behind this is not yet well-established. Although the spatially flat $\Lambda$ CDM model ${ }^{1}$ (Peebles 1984) is consistent with most observations (see e.g. Farooq et al. 2017; Scolnic et al. 2018; Planck Collaboration 2020; eBOSS Collaboration 2021), some potential observational discrepancies and theoretical puzzles (see e.g. Di Valentino et al. 2021a; Perivolaropoulos \& Skara 2021) suggest that there still is room for other cosmological models, including, for example, non-flat $\Lambda \mathrm{CDM}$ (the Planck Collaboration 2020 cosmic microwave background $(\mathrm{CMB})$ anisotropy $\mathrm{TT}, \mathrm{TE}, \mathrm{EE}+$ lowE + lensing data favor positive spatial curvature) as well as dynamical dark energy. These discrepancies and puzzles motivate us to also

\footnotetext{
* E-mail: shulei@phys.ksu.edu

$\dagger$ E-mail: jwryan@mail.smu.edu

$\ddagger$ E-mail: ratra@phys.ksu.edu

1 The flat $\Lambda$ CDM model has flat spatial hypersurfaces and a time-independent dark energy, a cosmological constant $\Lambda$, that provides approximately $70 \%$ of the current cosmological energy budget. Non-relativistic cold dark matter (CDM) accounts for approximately $25 \%$ and non-relativistic baryonic matter accounts for almost all of the remaining $\sim 5 \%$ of the energy budget.
}

study dynamical dark energy models and spatially non-flat models in this paper.

In this paper we use the new González-Morán et al. (2021) H II starburst galaxy (HIIG) measured fluxes and inferred absolute luminosities (from their correlation with their measured ionized gas velocity dispersions) as standard candles to constrain cosmological models. ${ }^{2}$ These new $\mathrm{HIIG}$ data reach to a slightly higher redshift $z \sim 2.5$, somewhat higher than the baryon acoustic oscillation (BAO) standard ruler data that reach to $z \sim 2.3$, that we also use in this paper. In order to determine the expansion rate and geometry of the Universe, it is vital to measure distances using either standard candles or standard rules. More data sets probing wider redshift regions would provide more information and make more contributions to a better understanding of our Universe, so it is worthwhile to seek additional stan-

\footnotetext{
2 Our analyses of the earlier González-Morán et al. (2019) data are described in Cao et al. (2020) (also see Cao et al. 2021a,b; Johnson et al. 2021). For analyses of earlier H IIG data, see Melnick et al. (2000); Siegel et al. (2005); Plionis et al. (2011); Mania \& Ratra (2012); Chávez et al. (2014); Terlevich et al. (2015); Chávez et al. (2016), and references therein. For recent analyses of the González-Morán et al. (2021) H IIG measurements, see Tsiapi et al. (2021); Mehrabi et al. (2022).
} 
dard rulers. The angular sizes of quasars (QSOs) provide one such additional probe, reaching to $z \sim 2.7$, which we have explored in previous work (Ryan et al. 2019; Cao et al. 2020, 2021a,b). As described in those papers and in Sec. 3 below, intermediate luminosity QSOs have, over a fairly wide range of redshifts $(0.46 \lesssim z \lesssim 2.7)$, very similar intrinsic lengths $l_{\mathrm{m}}=11.03 \pm 0.25 \mathrm{pc}$ (Cao et al. 2017). A knowledge of this intrinsic length scale, combined with measurements of the angular sizes of these QSOs allows one to determine the angular diameter distance out to the redshifts of the QSOs.

The QSO data that we have used in the past (from Cao et al. 2017) have the following drawback, however: $l_{\mathrm{m}}$ was determined with a Gaussian process interpolation (Seikel et al. 2012) of the Hubble parameter from Hubble parameter $(H(z))$ data (as described in Cao et al. 2017), many of which we have used in our previous analyses and also use in this paper. We have discussed this correlation between our QSO data and our $H(z)$ data in the past (Ryan 2021; Cao et al. 2021b), although we made the assumption in our earlier analyses that the correlation is not significant enough to have a strong effect on our results (owing to the weakness of the constraints from QSO data). Here we sidestep this problem by treating $l_{\mathrm{m}}$ as a free (nuisance) parameter, thereby constraining its value directly from our analysis. As discussed in Sec. 5 , we find that the value of $l_{\mathrm{m}}$ is almost independent of cosmological model, and is consistent with the value $l_{\mathrm{m}}=11.03 \pm 0.25 \mathrm{pc}$ from Cao et al. (2017) that we used in our earlier work. This finding suggests that these QSOs are close to being standard rulers, and it validates the result of Cao et al. (2017), independently of their method.

Significant constraints on cosmology now largely come from only a few data sets, at low $z \lesssim 2.3$, including BAO, Type Ia supernova (SN Ia), and $H(z)$ measurements, and at $z \sim 1100$ from CMB anisotropy observations. As mentioned above, it is useful and important to develop new probes, especially in the intermediate $2.3 \lesssim z \lesssim 1100$ redshift range. $\mathrm{H}$ IIG is an example, as are QSO angular sizes that have been under discussion for a longer time (see e.g. Gurvits et al. 1999; Vishwakarma 2001; Lima \& Alcaniz 2002; Zhu \& Fujimoto 2002; Chen \& Ratra 2003) with the compilation of Cao et al. (2017) being a significant step forward. Other probes under development now include reverberationmeasured $\mathrm{Mg}$ II time-lag radius-luminosity relation QSOs that reach to $z \sim 1.9$ (Martínez-Aldama et al. 2019; Czerny et al. 2021; Zajaček et al. 2021; Yu et al. 2021; Khadka et al. 2021a). High redshift options include QSO X-ray and UV flux measurements which extend to $z \sim 7.5$ (Risaliti \& Lusso 2015, 2019; Khadka \& Ratra 2020a,b, 2021a,b; Yang et al. 2020; Lusso et al. 2020; Li et al. 2021; Lian et al. 2021), ${ }^{3}$ and gamma-ray burst (GRB) data that extend to $z \sim 8.2$ (Amati et al. 2008, 2019; Samushia \& Ratra 2010; Wang et al. 2016; Demianski et al. 2021; Fana Dirirsa et al. 2019; Khadka \& Ratra 2020c; Khadka et al. 2021b; Wang et al. 2021; Hu et al. 2021). ${ }^{4}$ As of now, all five of these probes provide mostly only weak cosmological constraints, but new

3 However the current QSO compilation is standardizable up to only $z \sim 1.5-1.7$ (Khadka \& Ratra 2021b,a).

${ }^{4}$ Only a smaller sample of 118 GRBs is reliable enough to be used for cosmological purposes, but include GRBs that probe to $z \sim 8.2$ (Khadka \& Ratra 2020c; Khadka et al. 2021b). data should yield tighter constraints that have the potential to soon usefully probe the largely unexplored $2 \lesssim z \lesssim 8$ part of cosmological redshift space.

Our comparisons here between the constraints from the new González-Morán et al. (2021) data and the old González-Morán et al. (2019) data show that the new data provide more restrictive constraints on most cosmological parameters. As noted above, QSO angular size data provide relatively cosmological model-independent estimates of $l_{\mathrm{m}}$. We find that the cosmological constraints from $H(z)$, BAO, SN Ia, QSO, and the new HiIG measurements are not mutually inconsistent, thus we combine them to provide more restrictive constraints on the cosmological and nuisance parameters. The almost model-independent summary constraints from this data combination are measurements of the Hubble constant, $H_{0}=69.7 \pm 1.2 \mathrm{~km} \mathrm{~s}^{-1} \mathrm{Mpc}^{-1}$, the nonrelativistic matter density parameter, $\Omega_{\mathrm{m}_{0}}=0.293 \pm 0.021$, and the QSO characteristic linear size, $l_{\mathrm{m}}=10.93 \pm 0.25 \mathrm{pc}$. The estimate of $H_{0}$ is in better agreement with the median statistics estimate of Chen \& Ratra (2011) $\left(H_{0}=68 \pm 2.8\right.$ $\mathrm{km} \mathrm{s}^{-1} \mathrm{Mpc}^{-1}$ ) than with the measurements of Planck Collaboration (2020) $\left(H_{0}=67.4 \pm 0.5 \mathrm{~km} \mathrm{~s}^{-1} \mathrm{Mpc}^{-1}\right)$ and Riess et al. (2021) $\left(H_{0}=73.2 \pm 1.3 \mathrm{~km} \mathrm{~s}^{-1} \mathrm{Mpc}^{-1}\right)$. Although the most-favored model is the spatially-flat $\Lambda \mathrm{CDM}$ model, there is room for some mild dark energy dynamics and a little non-zero spatial curvature energy density. We also find that currently accelerating cosmological expansion is favored by most of the data combinations we study (except for QSO data alone).

This paper is organized as follows. The models we study are briefly described in Section 2. The data we used are introduced in Section 3 with the data analysis method presented in Section 4. We summarize our results and conclusions in Sections 5 and 6.

\section{COSMOLOGICAL MODELS}

We use various combinations of observational data to constrain the cosmological parameters of six spatially-flat and non-flat $\Lambda \mathrm{CDM}, \mathrm{XCDM}$, and $\phi \mathrm{CDM}$ models and study the goodness of fit. ${ }^{5}$ The main features of the models we use are summarized below. We assume a minimal neutrino sector, with three massless neutrino species, with the effective number of relativistic neutrino species $N_{\text {eff }}=3.046$. We neglect the late-time contribution of non-relativistic neutrinos and treat the baryonic $\left(\Omega_{\mathrm{b}_{0}} h^{2}\right)$ and cold dark matter $\left(\Omega_{\mathrm{c}_{0}} h^{2}\right)$ energy density parameters as free cosmological parameters to be determined from the data. The non-relativistic matter density parameter $\Omega_{\mathrm{m}_{0}}=\left(\Omega_{\mathrm{b}_{0}} h^{2}+\Omega_{\mathrm{c}_{0}} h^{2}\right) / h^{2}$ is a derived parameter.

In the $\Lambda \mathrm{CDM}$ models, the expansion rate function

5 For recent observational constraints on spatial curvature see Farooq et al. (2015), Chen et al. (2016), Rana et al. (2017), Ooba et al. (2018a,c), Yu et al. (2018), Park \& Ratra (2019c,a), Wei (2018), DES Collaboration (2019a), Li et al. (2020), Handley (2019), Efstathiou \& Gratton (2020), Di Valentino et al. (2021b), Velasquez-Toribio \& Fabris (2020), Vagnozzi et al. (2021a,b), KiDS Collaboration (2021), Arjona \& Nesseris (2021), Dhawan et al. (2021), and references therein. 
$E(z) \equiv H(z) / H_{0}$ as a function of redshift $z$ is

$E(z)=\sqrt{\Omega_{\mathrm{m}_{0}}(1+z)^{3}+\Omega_{\mathrm{k}_{0}}(1+z)^{2}+\Omega_{\Lambda}}$,

where

$\Omega_{\Lambda}=1-\Omega_{\mathrm{m}_{0}}-\Omega_{\mathrm{k}_{0}}$,

with $\Omega_{\mathrm{k}_{0}}$ being the curvature energy density parameter. There are four free parameters: $h, \Omega_{\mathrm{b}_{0}} h^{2}, \Omega_{\mathrm{c}_{0}} h^{2}$, and $\Omega_{\mathrm{k}_{0}}$ in the non-flat $\Lambda \mathrm{CDM}$ case and three in the flat case where $\Omega_{\mathrm{k}_{0}}=0$.

In the XCDM parametrizations, the expansion rate function is

$E(z)=\sqrt{\Omega_{\mathrm{m}_{0}}(1+z)^{3}+\Omega_{\mathrm{k}_{0}}(1+z)^{2}+\Omega_{\mathrm{X}_{0}}(1+z)^{3\left(1+w_{\mathrm{X}}\right)}}$,

where $w_{\mathrm{X}}$ is the equation of state parameter of the $\mathrm{X}$-fluid, and

$\Omega_{\mathrm{X}_{0}}=1-\Omega_{\mathrm{m}_{0}}-\Omega_{\mathrm{k}_{0}}$.

There are five free parameters: $h, \Omega_{\mathrm{b}_{0}} h^{2}, \Omega_{\mathrm{c}_{0}} h^{2}, \Omega_{\mathrm{k}_{0}}$, and $w_{\mathrm{X}}$ in the non-flat XCDM case and four in the flat case where $\Omega_{\mathrm{k}_{0}}=0$.

In the $\phi$ CDM models (Peebles \& Ratra 1988; Ratra $\&$ Peebles 1988; Pavlov et al. 2013), ${ }^{6}$ the expansion rate function is

$E(z)=\sqrt{\Omega_{\mathrm{m}_{0}}(1+z)^{3}+\Omega_{\mathrm{k}_{0}}(1+z)^{2}+\Omega_{\phi}(z, \alpha)}$,

where the energy density parameter of the scalar field $\phi$, $\Omega_{\phi}(z, \alpha)$, is determined by simultaneously numerically integrating the scalar field's equation of motion

$\ddot{\phi}+3\left(\frac{\dot{a}}{a}\right) \dot{\phi}+V^{\prime}(\phi)=0$,

with a potential energy density

$V(\phi)=\frac{1}{2} \kappa m_{p}^{2} \phi^{-\alpha}$,

and the Friedmann equation (5) where $H_{0} E(z)=\dot{a} / a$. In these equations $a$ is the scale factor and an overdot denotes a time derivative, the prime denotes a derivative with respect to the argument, $m_{p}$ is the Planck mass, the parameter $\alpha \geq$ 0 , and the constant $\kappa$ can be treated as a shooting parameter which is determined by the shooting method implemented in the Cosmic Linear Anisotropy Solving System (CLASS) code (Blas et al. 2011). There are five free parameters: $h, \Omega_{\mathrm{b}_{0}} h^{2}$, $\Omega_{\mathrm{c}_{0}} h^{2}, \Omega_{\mathrm{k}_{0}}$, and $\alpha$ in the non-flat $\phi$ CDM case and four in the flat case where $\Omega_{\mathrm{k}_{0}}=0$.

\section{DATA}

In this paper our main focus is on a new set of $\mathrm{HIIG}$ data (González-Morán et al. 2021, which we dub "H IIG-2021"). We compare cosmological constraints from these H IIG-2021

6 For recent observational constraints on the $\phi$ CDM model see Avsajanishvili et al. (2015), Solà Peracaula et al. (2018, 2019), Zhai et al. (2017), Ooba et al. (2018b, 2019), Park \& Ratra (2018, 2019b, 2020), Sangwan et al. (2018), Singh et al. (2019), UreñaLópez \& Roy (2020), Sinha \& Banerjee (2021), and references therein. data to those from earlier HiIG data. We also use these H IIG-2021 data and BAO, H(z), SN Ia, and QSO angular size measurements to constrain cosmological parameters in the models we study.

The $31 H(z)$ measurements we use, that span the redshift range $0.070 \leq z \leq 1.965$, are given in Table 2 of Ryan et al. (2018). ${ }^{7}$ The $11 \mathrm{BAO}$ measurements we use, that span the redshift range $0.38 \leq z \leq 2.334$, are listed in Table 1 of Cao et al. (2021b). ${ }^{8}$ Information on systematic errors of these data can be found in Cao et al. (2021a).

The SN-Pantheon data we use consist of $1048 \mathrm{SN}$ Ia measurements, spanning the redshift range $0.01<z<2.3$, compiled in Scolnic et al. (2018). The SN-DES data we use consist of 20 binned measurements (of 207 SN Ia measurements), spanning the redshift range $0.015 \leq z \leq 0.7026$, compiled in DES Collaboration (2019c). See Cao et al. (2021b) for a description of how we use these SN Ia data.

The QSO data we use, that span the redshift range $0.462 \leq z \leq 2.73$, are listed in Table 1 of Cao et al. (2017). These consist of 120 measurements of the angular size

$\theta(z)=\frac{l_{\mathrm{m}}}{D_{A}(z)}$.

Here $l_{\mathrm{m}}$ is the characteristic linear size of QSOs in the sample and $D_{A}$ (defined below) is the angular size distance. Here we improve on the approach of Cao et al. (2017), Ryan et al. (2019), and Cao et al. (2020, 2021a,b), by treating $l_{\mathrm{m}}$ as a nuisance parameter to be determined from these measurements so that these QSO data are independent of $H(z)$ data.

The old HiIG data (which we dub "HiIG-2019") consist of 107 low redshift measurements that span $0.0088 \leq$ $z \leq 0.16417$, used in Chávez et al. (2014) (recalibrated by González-Morán et al. 2019), and 46 high redshift measurements that $\operatorname{span} 0.636427 \leq z \leq 2.42935$. The new $\mathrm{H}_{\text {IIG- }}$ 2021 data, comprising the original 107 low redshift measurements and 74 updated high redshift measurements (that now span $0.636427 \leq z \leq 2.545$ ), are listed in Table A3 of González-Morán et al. (2021).

The correlation between $\mathrm{HIIG}$ luminosity $(L)$ and velocity dispersion $(\sigma)$ is

$\log L=\beta \log \sigma+\gamma$,

where $\beta$ and $\gamma$ are the slope and intercept. $\log =\log _{10}$ is implied everywhere. Both $\mathrm{H}$ IIG data sets are corrected for extinction by using the Gordon et al. (2003) extinction law, with

$\beta=5.022 \pm 0.058$

and

$\gamma=33.268 \pm 0.083$.

A detailed description of how to use $\mathrm{H}$ IIG data can be found in Cao et al. (2020). Note that the systematic uncertainties

7 These measurements were taken from Simon et al. (2005), Stern et al. (2010), Moresco et al. (2012), Zhang et al. (2014), Moresco (2015), Moresco et al. (2016), and Ratsimbazafy et al. (2017).

8 These measurements were taken from Alam et al. (2017), Ata et al. (2018), Carter et al. (2018), DES Collaboration (2019b), and du Mas des Bourboux et al. (2020). 
of both H HIG and QSO data are not considered so that the reduced $\chi^{2}$ 's are relatively large.

The transverse comoving distance $D_{M}(z)$, the luminosity distance $D_{L}(z)$, and the angular size distance $D_{A}(z)$ are related through $D_{M}(z)=D_{L}(z) /(1+z)=(1+z) D_{A}(z)$.

$$
D_{M}(z)= \begin{cases}D_{C}(z) & \text { if } \Omega_{\mathrm{k}_{0}}=0, \\ \frac{c}{H_{0} \sqrt{\Omega_{\mathrm{k}_{0}}}} \sinh \left[\sqrt{\Omega_{\mathrm{k}_{0}}} H_{0} D_{C}(z) / c\right] & \text { if } \Omega_{\mathrm{k}_{0}}>0, \\ \frac{c}{H_{0} \sqrt{\left|\Omega_{\mathrm{k}_{0}}\right|}} \sin \left[\sqrt{\left|\Omega_{\mathrm{k}_{0}}\right|} H_{0} D_{C}(z) / c\right] & \text { if } \Omega_{\mathrm{k}_{0}}<0\end{cases}
$$

where

$D_{C}(z) \equiv c \int_{0}^{z} \frac{d z^{\prime}}{H\left(z^{\prime}\right)}$

with $c$ being the speed of light (Hogg 1999).

\section{DATA ANALYSIS METHODOLOGY}

In this paper we use the ClASS code to compute cosmological model predictions as a function of the cosmological model and other parameters. These predictions are compared to observational data using the Markov chain Monte Carlo (MCMC) code MontePython (Audren et al. 2013) to maximize the likelihood function, $\mathcal{L}$, and thereby determine the best-fitting values of the free parameters. The priors on the cosmological parameters are flat and nonzero over the same ranges as used in Cao et al. (2021b), except that now $\Omega_{\mathrm{b}_{0}} h^{2} \in[0.00499,0.03993] .{ }^{9}$ The prior range of the QSO nuisance parameter $l_{\mathrm{m}}$ is not bounded.

The computation of the likelihood functions of $H(z)$, BAO, H IIG, and QSO data are described in Cao et al. (2020) and Cao et al. (2021a), whereas that of the likelihood functions of SN Ia measurements can be found in Cao et al. (2021b). One can also find the definitions of the Akaike Information Criterion $(A I C)$ and the Bayesian Information Criterion $(B I C)$ in those papers.

\section{RESULTS}

The posterior one-dimensional (1D) probability distributions and two-dimensional (2D) confidence regions of the cosmological and nuisance parameters for the six flat and non-flat models are shown in Figs. 1-6, in gray (QSO), pink (H IIG-2019), green (HiIG-2021), blue $(H(z)+$ BAO), red $(H(z)+\mathrm{BAO}+\mathrm{SN}-\mathrm{Pantheon}+\mathrm{SN}-\mathrm{DES}, \mathrm{HzBSNPD})$, and purple $(H(z)+\mathrm{BAO}+\mathrm{SN}-$ Pantheon + SN-DES + QSO + HiIG-2021, HzBSNPDQH). We list the unmarginalized best-fitting parameter values, as well as the corresponding $\chi^{2}, A I C, B I C$, degrees of freedom $\nu(\nu \equiv N-n)$, reduced $\chi^{2}\left(\chi^{2} / \nu\right), \Delta \chi^{2}, \Delta A I C$, and $\Delta B I C$ for all models and data

9 The value of primordial Helium abundance $Y_{\mathrm{p}}$ is set using a standard big-bang nucleosynthesis prediction by interpolation on a grid of values computed using version 1.2 of the PArthENoPE BBN code for a neutron lifetime of $880.2 \mathrm{~s}$. Since we choose the effective number of relativistic neutrino species $N_{\text {eff }}=3.046, \Omega_{\mathrm{b}_{0}} h^{2}$ is therefore limited to the range of $[0.00499,0.03993]$ by the correlated predictions of $Y_{\mathrm{p}}$. combinations, in Table 1. The marginalized posterior mean parameter values and uncertainties $( \pm 1 \sigma$ error bars or $2 \sigma$ limits), for all models and data combinations, are listed in Table $2 .^{10}$

\subsection{Constraints from $\mathrm{H}$ IIG-2021 versus H IIG-2019}

Here we compare constraints from the current compilation of H IIG data (H IIG-2021) with the constraints from the older compilation (H IIG-2019).

For both data sets, from the figures, most of the probability lies in the part of parameter space that corresponds to currently accelerating cosmological expansion.

For both the flat and non-flat $\Lambda$ CDM models, HiIG2021 data favor lower values of $\Omega_{\mathrm{c}_{0}} h^{2}$ and $\Omega_{\mathrm{m}_{0}}$. For the non-flat case, the HiIG-2021 data prefer a lower (more negative) value of $\Omega_{\mathrm{k}_{0}}$ than the HIIG-2019 data. It is worth noting that $H_{0}$ is not particularly sensitive to the change in the HiIG data. It is also worth noting that the HiIG2021 compilation provides constraints on $\Omega_{\mathrm{b}_{0}} h^{2}$ in the flat case, in contrast to $\mathrm{H} I \mathrm{IG}-2019$, although these constraints are not as tight as those obtained from the other data combinations. H IIG-2021 data also more tightly constrain $\Omega_{\mathrm{k}_{0}}$ compared to the HiIG-2019 data. In comparison with the flat $\Lambda$ CDM H IIG-2021 constraints given in González-Morán et al. (2021), $h=0.717 \pm 0.018$ and $\Omega_{\mathrm{m}_{0}}=0.243_{-0.050}^{+0.039}$, our constraints, $h=0.7191 \pm 0.0192$ and $\Omega_{\mathrm{m}_{0}}=0.243_{-0.051}^{+0.039}$, are a bit less restrictive (due to our models having more free parameters) but are consistent with theirs.

In the flat and non-flat XCDM parametrizations, the H IIG-2021 data favor lower values of $\Omega_{\mathrm{c}_{0}} h^{2}, \Omega_{\mathrm{m}_{0}}, w_{\mathrm{X}}$, and $\Omega_{\mathrm{k}_{0}}$ than those favored by the HilG-2019 data, while the constraints on $\Omega_{\mathrm{b}_{0}} h^{2}$ and $H_{0}$ are consistent with each other. The flat XCDM HiIG-2021 constraints are $\left\{h, \Omega_{\mathrm{m}_{0}}, w_{\mathrm{X}}\right\}=$ $\left\{0.719 \pm 0.020,0.250_{-0.061}^{+0.10},-1.19_{-0.38}^{+0.46}\right\}$ in González-Morán et al. (2021), whereas our results are $\left\{h, \Omega_{\mathrm{m}_{0}}, w_{\mathrm{X}}\right\}=$ $\left\{0.7266 \pm 0.0219,0.288_{-0.058}^{+0.087},-1.527_{-0.391}^{+0.786}\right\}$ and consistent with theirs within $1 \sigma$, although due to different prior ranges the posterior means deviate more than those for the flat $\Lambda \mathrm{CDM}$ model.

In the flat $\phi \mathrm{CDM}$ model, the HiIG-2021 data prefer lower values of $\Omega_{\mathrm{c}_{0}} h^{2}, \Omega_{\mathrm{m}_{0}}$, and $\alpha$, with consistent constraints on $\Omega_{\mathrm{b}_{0}} h^{2}$ and $H_{0}$. The HiIG-2021 data constrain $\alpha$ more tightly than the $\mathrm{H}$ IIG-2019 data, leading to $\alpha$ being consistent with zero to within a little more than $1 \sigma$. In the non-flat case, the $\mathrm{H}$ IIG-2021 data prefer lower values for all parameters. It is worth noting that both $\mathrm{H}_{\mathrm{IIG}} \mathrm{-2021}$ and $\mathrm{H}_{\mathrm{IIG}} \mathrm{-2019}$ data in the flat and non-flat $\phi \mathrm{CDM}$ models determine lower values of $\Omega_{\mathrm{m}_{0}}$, and $\mathrm{HIIG}_{\mathrm{II}} 2021$ data prefer the lowest $\Omega_{\mathrm{m}_{0}}$ value in the non-flat $\phi \mathrm{CDM}$ model among all models.

HIIG-2021 and HiIG-2019 data result in higher values of $H_{0}$ than the other probes we study in this paper. The highest $H_{0}$ values are in the flat XCDM parametrization and are $72.66 \pm 2.19 \mathrm{~km} \mathrm{~s}^{-1} \mathrm{Mpc}^{-1}$ and $72.37_{-2.20}^{+2.18}$ $\mathrm{km} \mathrm{s}^{-1} \mathrm{Mpc}^{-1}$, respectively, which are $1.31 \sigma$ and $1.23 \sigma$ higher than the median statistics estimate of $H_{0}=68 \pm 2.8$

10 We use the Python package GetDist (Lewis 2019) to determine the posterior means and uncertainties and to generate the marginalized likelihood contours. 


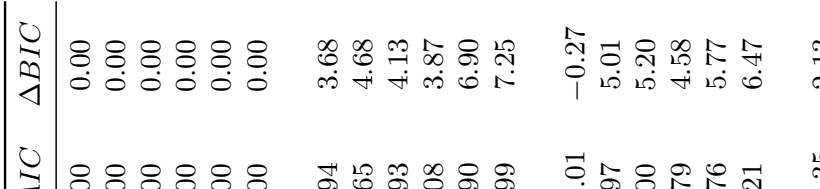

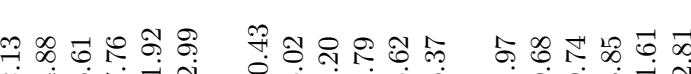

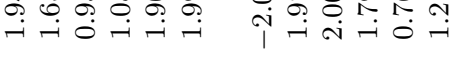

น้ำ 국

둥 $85 \overrightarrow{0}=$

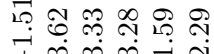

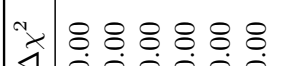

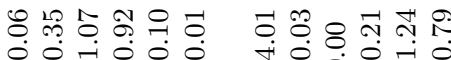

남요용ㅇㅇㅇㅛ 음

|

$\triangleleft$

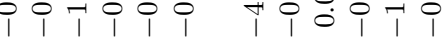

1

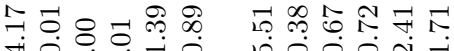

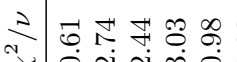

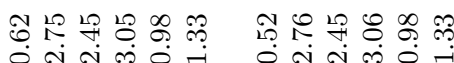

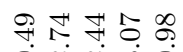

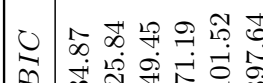

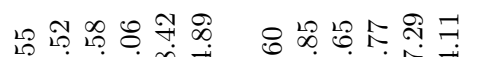

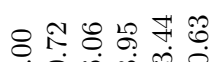

1 10007

Lि

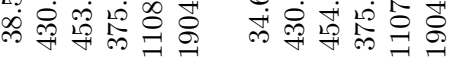

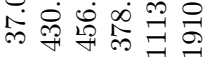

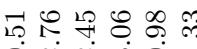

완ㄷㄴ 유요

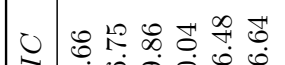

우와웜유

เฉ

-

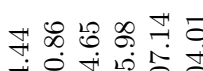

○ त त

丩

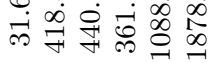

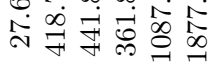

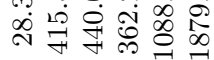

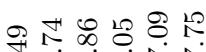

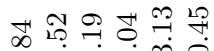

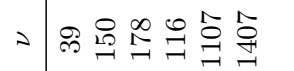

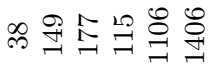

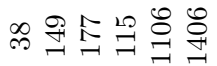

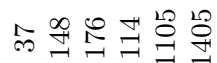

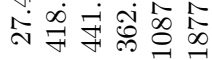

능ำ

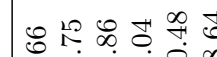

으유 요구

ถึำ

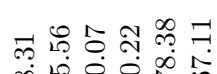

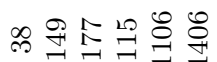

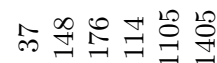

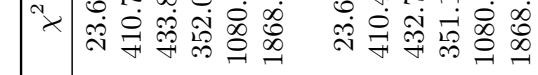

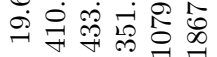

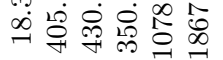

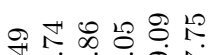

เूล

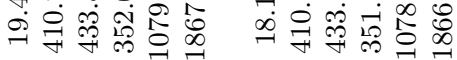

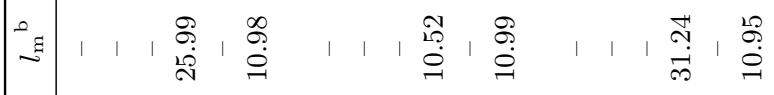

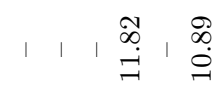

兽, 葛

के,

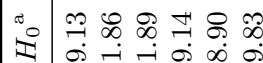

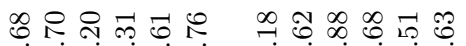

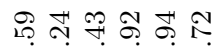

平只

욕

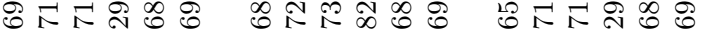

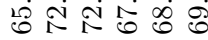

मिं

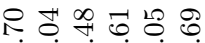

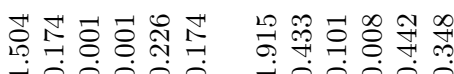

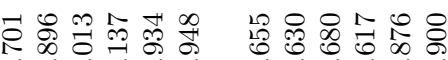

ili i i

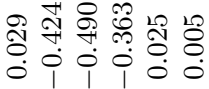

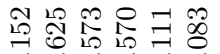

i

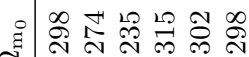

귱

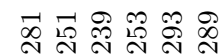

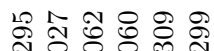

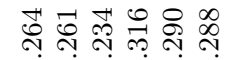

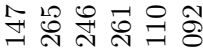

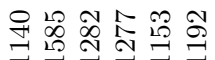
(1)

000000

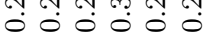

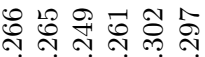

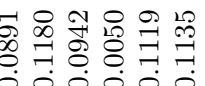

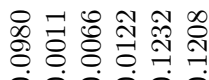

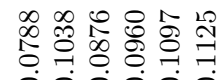

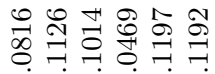
000000

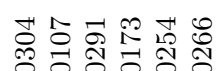

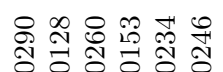

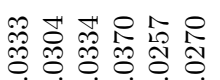

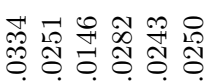
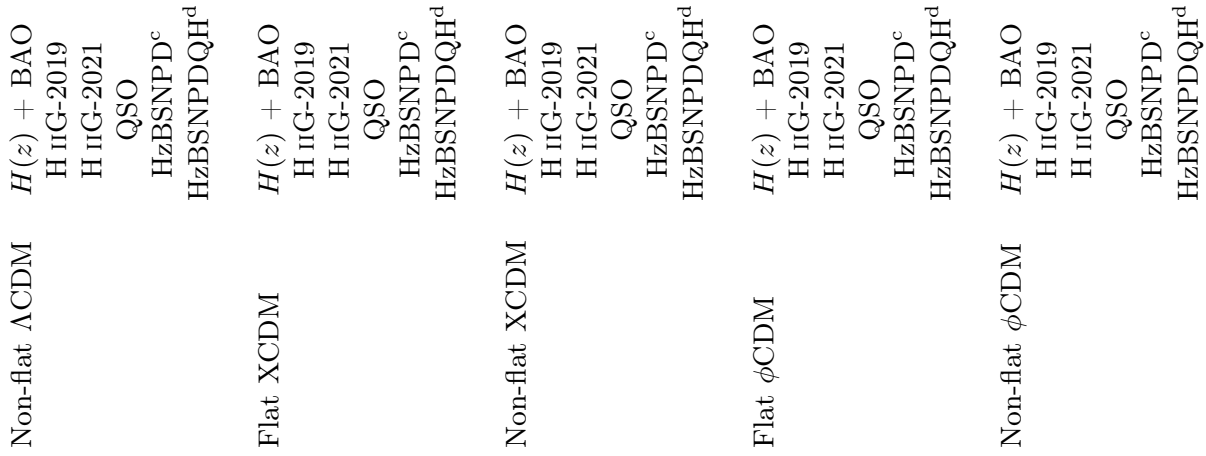

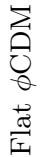

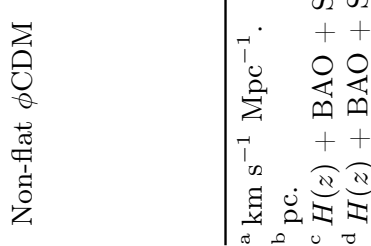


$\mathrm{km} \mathrm{s}^{-1} \mathrm{Mpc}^{-1}$ (Chen \& Ratra 2011), and $0.21 \sigma$ and $0.33 \sigma$ lower than the local Hubble constant measurement of $H_{0}=$ $73.2 \pm 1.3 \mathrm{~km} \mathrm{~s}^{-1} \mathrm{Mpc}^{-1}$ (Riess et al. 2021). The lowest $H_{0}$ estimates are in the non-flat $\phi \mathrm{CDM}$ model and are $70.49 \pm 1.81 \mathrm{~km} \mathrm{~s}^{-1} \mathrm{Mpc}^{-1}$ and $70.53 \pm 1.79 \mathrm{~km} \mathrm{~s}^{-1} \mathrm{Mpc}^{-1}$, which are $0.75 \sigma$ and $0.76 \sigma$ higher than the median statistics estimate of $H_{0}=68 \pm 2.8 \mathrm{~km} \mathrm{~s}^{-1} \mathrm{Mpc}^{-1}$, and $1.22 \sigma$ and $1.21 \sigma$ lower than the local Hubble constant measurement of $H_{0}=73.2 \pm 1.3 \mathrm{~km} \mathrm{~s}^{-1} \mathrm{Mpc}^{-1}$.

In the non-flat $\Lambda$ CDM model, $\mathrm{HIIG}_{\mathrm{II}} 2021$ and $\mathrm{HIIG}$ 2019 data favor closed spatial hypersurfaces, while in the non-flat XCDM parametrization and the non-flat $\phi \mathrm{CDM}$ model, they favor open spatial hypersurfaces. Only in the non-flat $\phi \mathrm{CDM}$ model, however, is $\Omega_{\mathrm{k}_{0}}$ more than $1 \sigma$ away from spatial flatness. Dark energy dynamics is favored by both data sets, but dark energy being a cosmological constant is not disfavored (it is within $1 \sigma$ or just a little bit more away).

\subsection{QSO constraints alone and in comparison to those from other probes}

In this subsection we discuss the constraints we obtain solely from the QSO data. As mentioned in Sec. 3, in this paper we improve on earlier analyses of the QSO angular size data by now treating $l_{\mathrm{m}}$, the characteristic linear size of QSOs, as a nuisance parameter to be determined from the observational data. From QSO data alone, in Table $2, l_{\mathrm{m}}$ ranges from a low of $10.26_{-3.42}^{+1.24} \mathrm{pc}$ for the non-flat $\phi \mathrm{CDM}$ model to a high of $11.90_{-4.17}^{+1.52} \mathrm{pc}$ for the flat XCDM parametrization, differing by just $0.38 \sigma$. These values are consistent from model to model, largely justifying the use of QSOs as standard rulers, with $l_{\mathrm{m}}=11.03 \mathrm{pc}$, the value we used in our previous studies (taken from Cao et al. 2017). However, ignoring the dependence on cosmological model and the $l_{\mathrm{m}}$ errors, as we and others have previously done, results in mildly biased and somewhat more restrictive QSO angular size constraints than is warranted by data. These deficiencies are corrected in our improved analyses here. ${ }^{11}$ Additionally, we note that in Table 1 , the best-fitting values of $H_{0}$ in flat $\Lambda$ CDM and flat XCDM appear to be unreasonably low. This strange behavior is caused by the large values of $l_{\mathrm{m}}$, which push the $H_{0}$ values lower to obtain locally minimized $\chi^{2}$ values. Specifically, from the form of the model-predicted angular size of a quasar,

$\theta(z) \propto \frac{l_{\mathrm{m}} H_{0}}{d_{\mathrm{M}}(z)}$

(where $d_{\mathrm{M}}(z):=\frac{H_{0}}{c} D_{\mathrm{M}}(z)$ and suppressing irrelevant parameters), we can see that a large value of $l_{\mathrm{m}}$ requires a small value of $H_{0}$ in order to keep $\theta(z)$ constant. Since $l_{\mathrm{m}}$ has an unbounded prior range, it can roam over a larger region of parameter space than $H_{0}$. It therefore has the freedom to move into regions of parameter space where its value is unusually large; if this happens, then $H_{0}$ must be made small to compensate. This is only a partial answer, since

11 When QSO data are combined with other probes, as in the HzBSNPDQH combination, the model-independence of $l_{\mathrm{m}}$ is evident and the determination here is consistent with $l_{\mathrm{m}}=11.03 \pm$ 0.25 pc found by Cao et al. (2017). it does not account for the variation of $\Omega_{\mathrm{k}_{0}}$ (the effect of which is more complex, as $\Omega_{\mathrm{k}_{0}}$ is coupled to the redshift $z$ through the function $d_{\mathrm{M}}(z)$ ), and so does not fully capture the behavior of $\theta(z)$ across all models, but it does give some insight into the apparently anomalously low values of $H_{0}$ that appear in some cases. ${ }^{12}$

From the results listed in Table 2, we can draw the following conclusions. First, QSO data alone can only constrain the values of $\Omega_{\mathrm{b}_{0}} h^{2}$ in the flat and non-flat $\phi$ CDM models. Second, QSO data alone prefer higher values of $\Omega_{\mathrm{m}_{0}}$, which are consistent with almost all other probes except for the non-flat $\phi$ CDM H IIG-2021 case (the posterior mean values being $1.1 \sigma$ away from each other in this case). Furthermore, QSO data alone do not give tight constraints on $H_{0}$ or $\Omega_{\mathrm{k}_{0}}$. Although in each non-flat model open geometry is favored, given the large error bars, flat geometry is within $1 \sigma$.

QSO data favor higher central values of $\Omega_{\mathrm{c}_{0}} h^{2}$ and $\Omega_{\mathrm{m}_{0}}$, in both flat and non-flat $\Lambda \mathrm{CDM}$, compared to the central values favored by the other probes (although QSO constraints have wider error bars than the other constraints). QSO data only very weakly constrain the value of $H_{0}$ in the flat $\Lambda$ CDM model, while the fit of QSO data to the non-flat $\Lambda \mathrm{CDM}$ model produces a tighter constraint whose central value is closer to that of the $\mathrm{HIIG}$ data and the local value favored by Riess et al. (2021) (with wide error bars, however). In both the flat and non-flat cases, the marginalized values of $l_{\mathrm{m}}$ are close to the value obtained by Cao et al. (2017), with the central value in the flat $\Lambda$ CDM model here being only $0.02 \mathrm{pc}$ away from that of Cao et al. (2017) (with wider error bars than what they found). QSO data do not provide strong evidence for non-zero spatial curvature in the non-flat $\Lambda$ CDM model, as the marginalized posterior mean value of $\Omega_{\mathrm{k}_{0}}$ is consistent with $\Omega_{\mathrm{k}_{0}}=0$ to within $1 \sigma$.

When we look at the flat and non-flat XCDM parametrizations, we find that QSO data again favor somewhat large values of $\Omega_{\mathrm{c}_{0}} h^{2}$ and $\Omega_{\mathrm{m}_{0}}$ (but, as with flat and non-flat $\Lambda$ CDM, these have wide error bars) and weak constraints on $H_{0}$. The central value of $H_{0}$ in the non-flat case is more consistent with Riess et al. (2021) and with the values derived from the $\mathrm{H}$ IIG data. In both cases we find that the marginalized values of $l_{\mathrm{m}}$ are consistent with that of Cao et al. (2017). We also find that QSO data favor values of $w_{\mathrm{X}}$ that are in the phantom regime (consistent with the findings from the HiIG data). In the non-flat case, QSO data favor a relatively large and positive central value (0.170) for $\Omega_{\mathrm{k}_{0}}$, corresponding to a spatially open universe, but the error bars are wide enough that this result is still consistent with spatial flatness.

Both the flat and non-flat $\phi \mathrm{CDM}$ models have central

12 The relatively higher values of $H_{0}$ seen in the $\phi$ CDM models pose an apparent challenge to this explanation, but here the bestfitting values of $\Omega_{\mathrm{b}_{0}} h^{2}$ and $\Omega_{\mathrm{c}_{0}} h^{2}$ need to be taken into account. In comparing, for example, the flat $\Lambda \mathrm{CDM}$ model to the flat $\phi \mathrm{CDM}$ model (both of which have nearly identical best-fitting values of $\Omega_{\mathrm{m}_{0}}$ ), we can see that the flat $\phi$ CDM model has larger best-fitting values of both $\Omega_{\mathrm{b}_{0}} h^{2}$ and $\Omega_{\mathrm{c}_{0}} h^{2}$. From the defining relationship $\Omega_{\mathrm{m}_{0}}=\left(\Omega_{\mathrm{b}_{0}} h^{2}+\Omega_{\mathrm{c}_{0}} h^{2}\right) / h^{2}$, keeping $\Omega_{\mathrm{m}_{0}}$ constant requires $\Omega_{\mathrm{b}_{0}} h^{2}$ $+\Omega_{\mathrm{c}_{0}} h^{2}$ and $H_{0}$ to vary in tandem. If $\Omega_{\mathrm{b}_{0}} h^{2}$ and $\Omega_{\mathrm{c}_{0}} h^{2}$ both increase, as they do in going from flat $\Lambda$ CDM to flat $\phi \mathrm{CDM}$, then $H_{0}$ must also increase. This then has the effect of lowering $l_{\mathrm{m}}$ (all other parameters being held fixed). 
values of $\Omega_{\mathrm{b}_{0}} h^{2}$ from QSO data that are similar to earlier findings (specifically, they are close to the values of $\Omega_{\mathrm{b}_{0}} h^{2}$ obtained for the flat $\Lambda \mathrm{CDM}$ and $\phi \mathrm{CDM}$ models by Park $\&$ Ratra 2018, 2019c). Both flat and non-flat $\phi$ CDM have relatively high central values of $\Omega_{\mathrm{c}_{0}} h^{2}$ and $\Omega_{\mathrm{m}_{0}}$ (compared to the other probes), both favor similar large values of $\alpha$ (consistent with $\alpha=0$, however, to within $1.15 \sigma$ and $1.29 \sigma$ in the flat and non-flat cases, respectively), and both show weak constraints on $H_{0}$. Both flat and non-flat $\phi \mathrm{CDM}$ favor posterior mean values of $l_{\mathrm{m}}$ that are consistent to within $1 \sigma$ with the central value obtained by Cao et al. (2017). Like non-flat XCDM, non-flat $\phi \mathrm{CDM}$ favors a relatively large and positive value of $\Omega_{\mathrm{k}_{0}}$, that is nevertheless consistent with spatial flatness to within $1 \sigma$.

\section{$5.3 \quad$ Joint analyses results}

Since the constraints derived from $H(z)$, BAO, SNPantheon, SN-DES, QSO, HiIG-2019, and HiIG-2021 data are not mutually inconsistent, we jointly analyze combinations of these data and summarize these results in this subsection.

The $H(z)+\mathrm{BAO}$ and HzBSNPD results are different from, but consistent with, what we obtained in Cao et al. (2021b). The differences arise from the different codes that we used to analyze the data; in Cao et al. (2021b) we used EMCEE, whereas here we used ClAss and MontePython. It is worth recalling here that, as mentioned above, CLASS constrains $\Omega_{\mathrm{b}_{0}} h^{2}$ in the range $0.00499 \leq \Omega_{\mathrm{b}_{0}} h^{2} \leq 0.03993$. Therefore the parameter constraints differ more when the model and data prefer higher values of $\Omega_{\mathrm{b}_{0}} h^{2}$; this is especially true of the $\phi \mathrm{CDM}$ model when it is fitted to the $H(z)+$ BAO data combination. As a result, the present constraints on $\Omega_{\mathrm{m}_{0}}$ and $\alpha$ in $\phi \mathrm{CDM}$ with $H(z)+\mathrm{BAO}$ data are higher and lower than the ones given in Cao et al. (2021b). The HzBSNPD results are, however, consistent.

The fit to the HzBSNPDQH data combination produces, for all models, the most interesting results. By adding QSO and HiIG-2021 data to HzBSNPD combination, the constraints are slightly tightened. Although the posterior means of $\Omega_{\mathrm{b}_{0}} h^{2}$ and $\Omega_{\mathrm{c}_{0}} h^{2}$ are relatively higher, those of $\Omega_{\mathrm{m}_{0}}$ are lower than the constraints from HzBSNPD. The $\Omega_{\mathrm{m}_{0}}$ constraints range from a low of $0.282 \pm 0.016$ (flat $\phi \mathrm{CDM}$ ) to a high of $0.298 \pm 0.013$ (flat $\Lambda \mathrm{CDM}$ ), a difference of only $0.78 \sigma$.

The constraints on $H_{0}$ are between $H_{0}=69.54 \pm$ $1.17 \mathrm{~km} \mathrm{~s}^{-1} \mathrm{Mpc}^{-1}$ (flat $\phi \mathrm{CDM}$ ) and $H_{0}=69.95 \pm 1.18$ $\mathrm{km} \mathrm{s}^{-1} \mathrm{Mpc}^{-1}$ (flat $\Lambda \mathrm{CDM}$ ) - a difference of only $0.25 \sigma$ — which are $0.64 \sigma$ (flat $\Lambda \mathrm{CDM}$ ) and $0.51 \sigma$ (flat $\phi \mathrm{CDM}$ ) higher than the median statistics estimate of $H_{0}=68 \pm$ $2.8 \mathrm{~km} \mathrm{~s}^{-1} \mathrm{Mpc}^{-1}$ (Chen \& Ratra 2011), and $1.85 \sigma$ (flat $\Lambda \mathrm{CDM}$ ) and $2.09 \sigma$ (flat $\phi \mathrm{CDM}$ ) lower than the local Hubble constant measurement of $H_{0}=73.2 \pm 1.3 \mathrm{~km} \mathrm{~s}^{-1} \mathrm{Mpc}^{-1}$ (Riess et al. 2021). ${ }^{13}$

For non-flat $\Lambda$ CDM, non-flat XCDM, and non-flat

13 Other local expansion rate $H_{0}$ measurements result in slightly lower central values with slightly larger error bars (Rigault et al. 2015; Zhang et al. 2017; Dhawan et al. 2018; Fernández Arenas et al. 2018; Breuval et al. 2020; Efstathiou 2020; Khetan et al. 2021; Rameez \& Sarkar 2021; Freedman 2021). Our $H_{0}$ estimates are consistent with earlier median statistics determi- $\phi \mathrm{CDM}$, we find $\Omega_{\mathrm{k}_{0}}=0.011 \pm 0.067, \Omega_{\mathrm{k}_{0}}=-0.054 \pm 0.096$, and $\Omega_{\mathrm{k}_{0}}=-0.072_{-0.073}^{+0.074}$, respectively. The non-flat XCDM and $\phi \mathrm{CDM}$ models favor closed geometry, while the non-flat $\Lambda \mathrm{CDM}$ model favors open geometry. Note, however, that these results are all consistent with spatially flat hypersurfaces to within $1 \sigma$.

Our results show a slight preference for dark energy dynamics. For flat (non-flat) XCDM, $w_{\mathrm{X}}=-0.950 \pm 0.062$ $\left(w_{\mathrm{X}}=-0.926_{-0.062}^{+0.091}\right)$, with central values being $0.81 \sigma$ $(1.19 \sigma)$ away from $w_{\mathrm{X}}=-1$; and for flat (non-flat) $\phi \mathrm{CDM}$, $\alpha=0.288_{-0.252}^{+0.098}\left(\alpha=0.405_{-0.304}^{+0.165}\right)$, with central values being $1.14 \sigma(1.33 \sigma)$ away from $\alpha=0$.

The constraints on the nuisance parameter $l_{\mathrm{m}}$ are between $l_{\mathrm{m}}=10.87 \pm 0.26 \mathrm{pc}$ (non-flat $\phi \mathrm{CDM}$ ) and $l_{\mathrm{m}}=$ $10.96 \pm 0.26 \mathrm{pc}$ (flat $\Lambda \mathrm{CDM}$ ), which differ by $0.24 \sigma$ and so are effectively model-independent, and consistent with $l_{\mathrm{m}}=11.03 \pm 0.25 \mathrm{pc}$ (Cao et al. 2017).

\subsection{Model comparison}

The values of the reduced $\chi^{2}\left(\chi^{2} / \nu\right), \Delta \chi^{2}, \Delta A I C$, and $\triangle B I C$ are reported in Table 1 , where $\Delta \chi^{2}, \Delta A I C$, and $\triangle B I C$, are the differences between the values of the $\chi^{2}$, $A I C$, and $B I C$ for a given model and the ones for flat $\Lambda C D M$. Here a negative (positive) value of $\Delta \chi^{2}, \Delta A I C$, or $\triangle B I C$ means that the given statistic favors (disfavors) the model under consideration relative to flat $\Lambda \mathrm{CDM}$. We find that, except for a few of the $H(z)+\mathrm{BAO}$ and $\mathrm{HiIG}-$ 2019 cases, the flat $\Lambda \mathrm{CDM}$ model is the most favored model among all six models we study. The $A I C$ does not show strong evidence against any of the models. ${ }^{14}$ However, we find that some data combinations show strong evidence against the models we study, when these models are analyzed using the BIC, as follows. First, the HzBSNPD combination strongly disfavors non-flat $\Lambda \mathrm{CDM}$ and very strongly disfavors non-flat XCDM and non-flat $\phi \mathrm{CDM}$. Second, the HzBSNPDQH combination strongly disfavors nonflat $\Lambda \mathrm{CDM}$, flat XCDM, and flat $\phi \mathrm{CDM}$, and very strongly disfavors non-flat XCDM and non-flat $\phi$ CDM. Furthermore, strong evidence against non-flat XCDM as well as non-flat $\phi \mathrm{CDM}$ are provided by the HIIG-2021 and QSO data.

\section{CONCLUSION}

We find that the new $\mathrm{H}$ IIG-2021 data provide more restrictive cosmological parameter constraints and also prefer lower values of $\Omega_{\mathrm{m}_{0}}, w_{\mathrm{X}}$, and $\Omega_{\mathrm{k}_{0}}$ than those favored by the $\mathrm{H}_{\mathrm{IIG}}$ 2019 data.

nations (Gott et al. 2001; Calabrese et al. 2012) as well as with other recent $H_{0}$ measurements (Chen et al. 2017; DES Collaboration 2018; Gómez-Valent \& Amendola 2018; Planck Collaboration 2020; Domínguez et al. 2019; Cuceu et al. 2019; Zeng \& Yan 2019; Schöneberg et al. 2019; Blum et al. 2020; Lyu et al. 2020; Philcox et al. 2020; Birrer et al. 2020; Denzel et al. 2021; Pogosian et al. 2020; Boruah et al. 2021; Kim et al. 2020; Harvey 2020; Zhang \& Huang 2021; Lin \& Ishak 2021).

14 There is weak evidence for the reference model when $\triangle A I C(B I C) \in[0,2]$, positive evidence when $\triangle A I C(B I C) \in$ $(2,6]$, strong evidence when $\triangle A I C(B I C) \in(6,10]$, and very strong evidence when $\triangle A I C(B I C)>10$ (Kass \& Raftery 1995). 


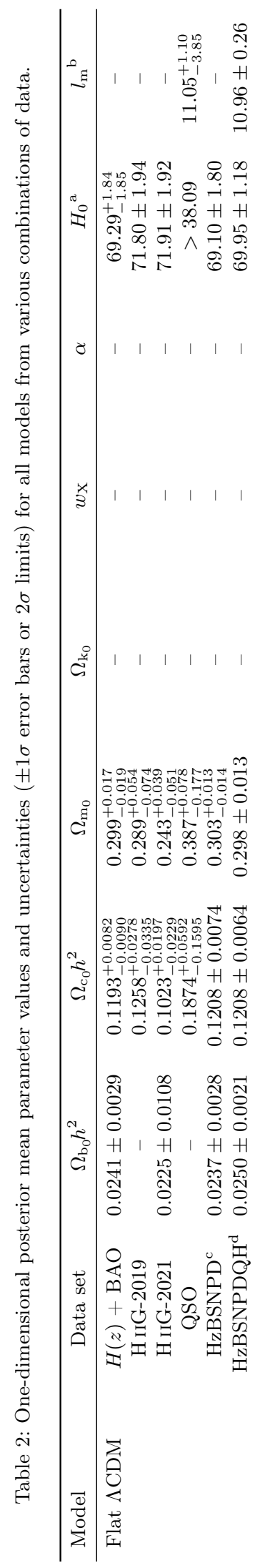

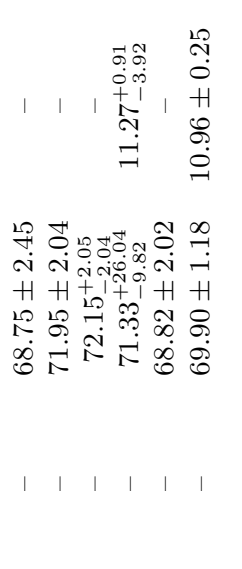

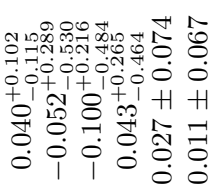

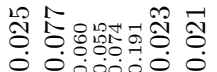
H H

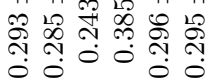
\&요에 0.000000000000

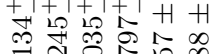

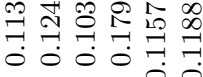

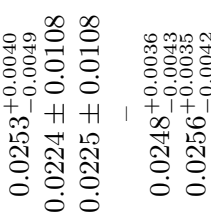

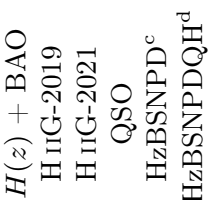

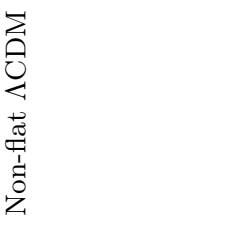

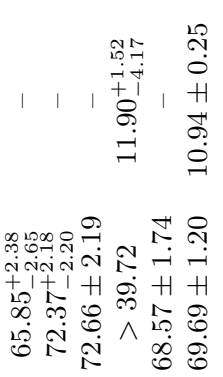

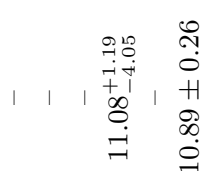

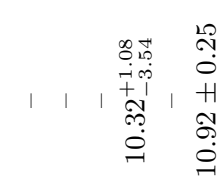

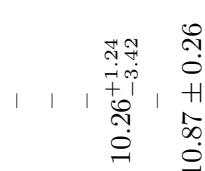

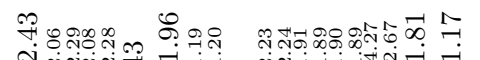

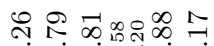

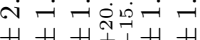

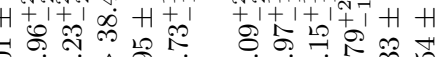

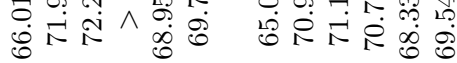

赤 政

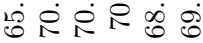

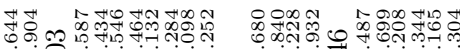
ón

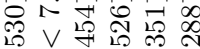

$+1+i 0+1+1+1$

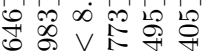

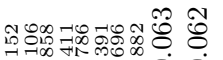

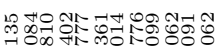
00000000 o 0000001000000

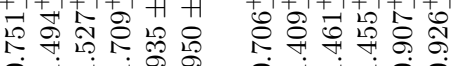

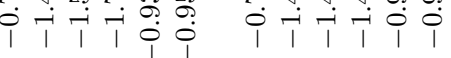

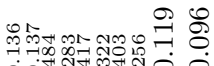
oionosion

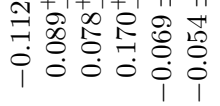

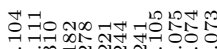

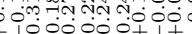

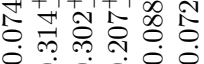

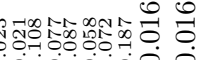
0
$+1+1+10 i+1$

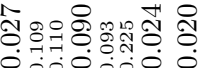

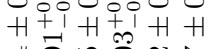

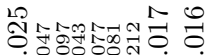

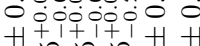

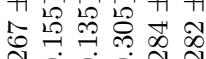
○ै

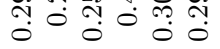

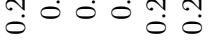

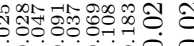

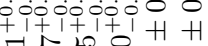
壳

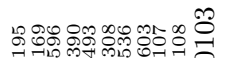

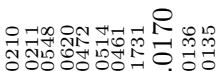
0.000000000000
$1+1+1+1+1$

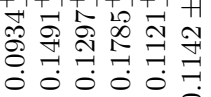

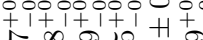

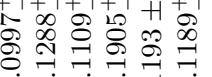

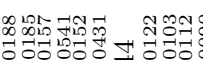

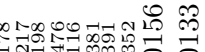

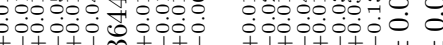

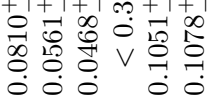

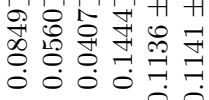

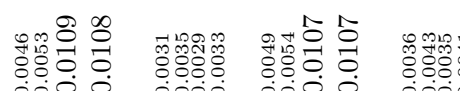
نं 0 .

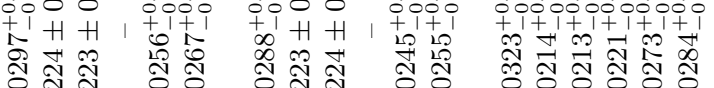

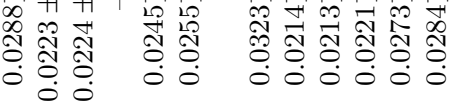

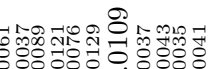

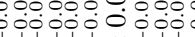

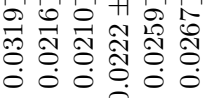

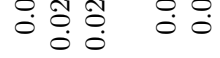

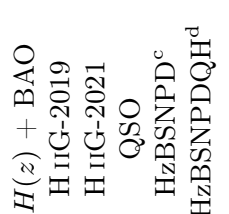

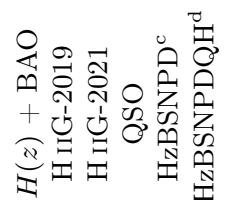

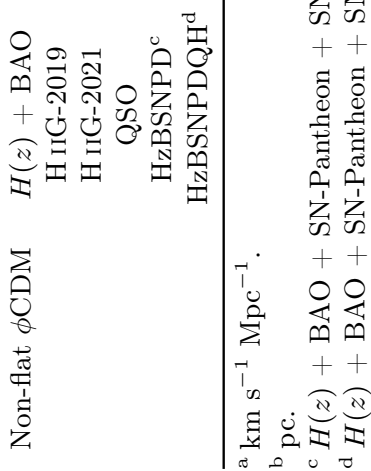




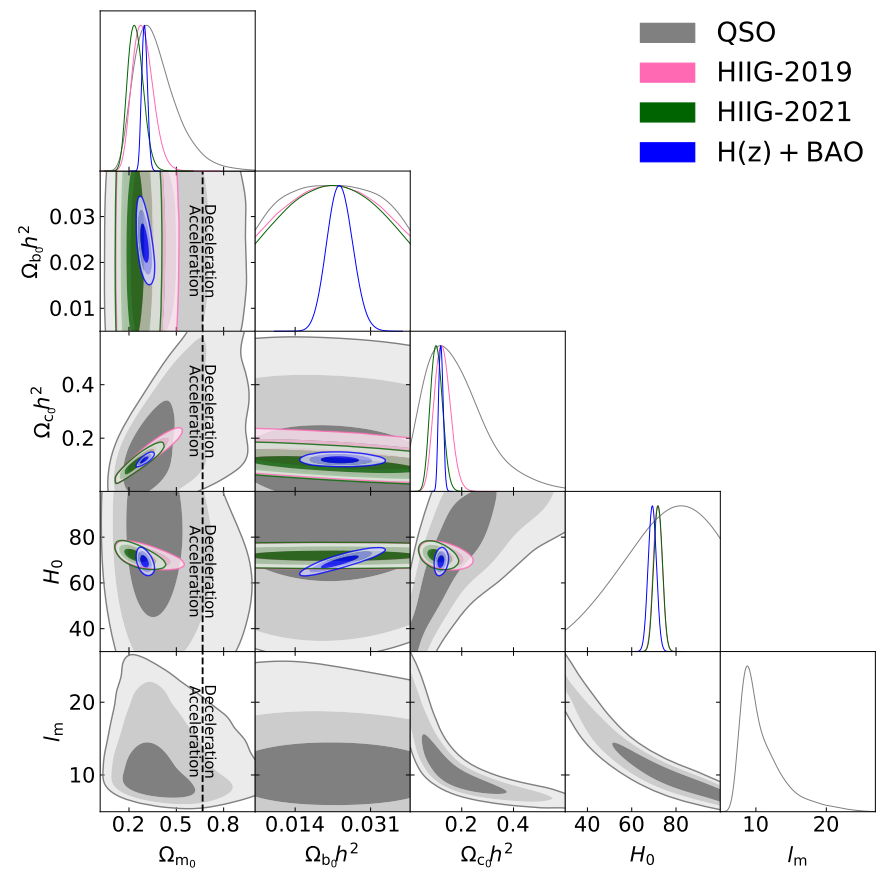

(a)

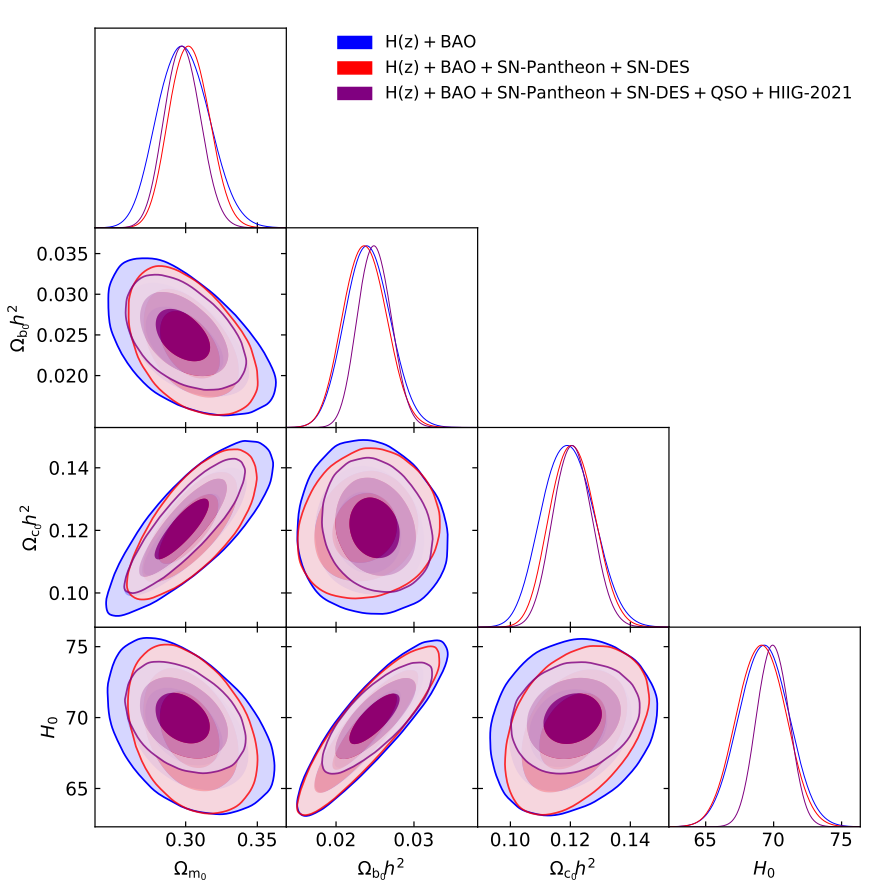

(b)

Figure 1. One-dimensional likelihoods and $1 \sigma, 2 \sigma$, and $3 \sigma$ two-dimensional likelihood confidence contours for flat $\Lambda$ CDM. Left panel shows individual data set and $H(z)+\mathrm{BAO}$ results and the right panel shows joint data sets constraints. The zero-acceleration lines are shown as black dashed lines in the left panel, which divide the parameter space into regions associated with currently-accelerating (left) and currently-decelerating (right) cosmological expansion, while in the right panel the joint analyses favor currently-accelerating expansion.

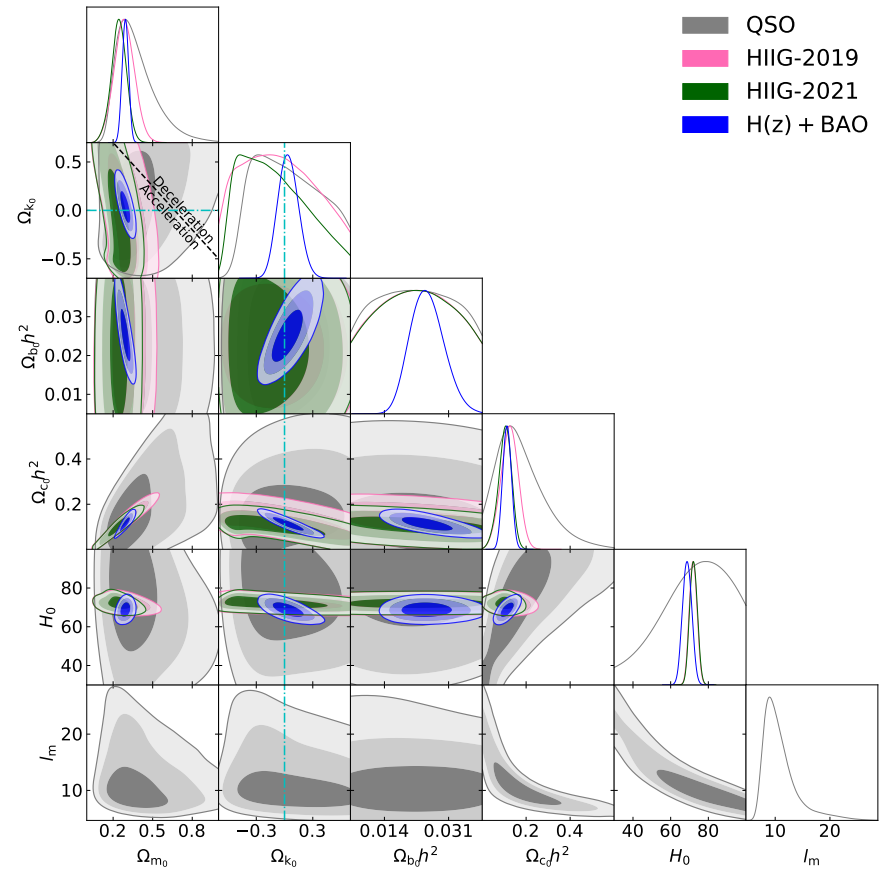

(a)

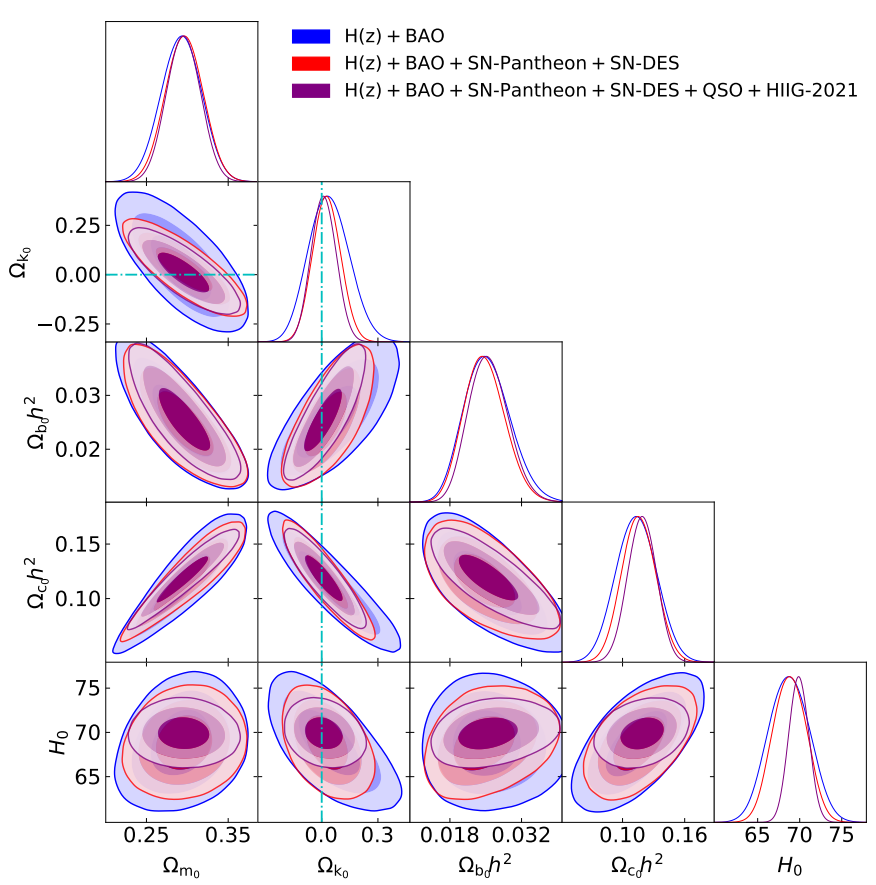

(b)

Figure 2. Same as Fig. 1 but for non-flat $\Lambda$ CDM. The flat $\Lambda$ CDM case is shown as the cyan dash-dot lines, with closed spatial hypersurfaces either below or to the left. The black dashed line in the left panel is the zero-acceleration line, which divides the parameter space into regions associated with currently-accelerating (below left) and currently-decelerating (above right) cosmological expansion. In the right panel, the joint analyses favor currently-accelerating expansion. 


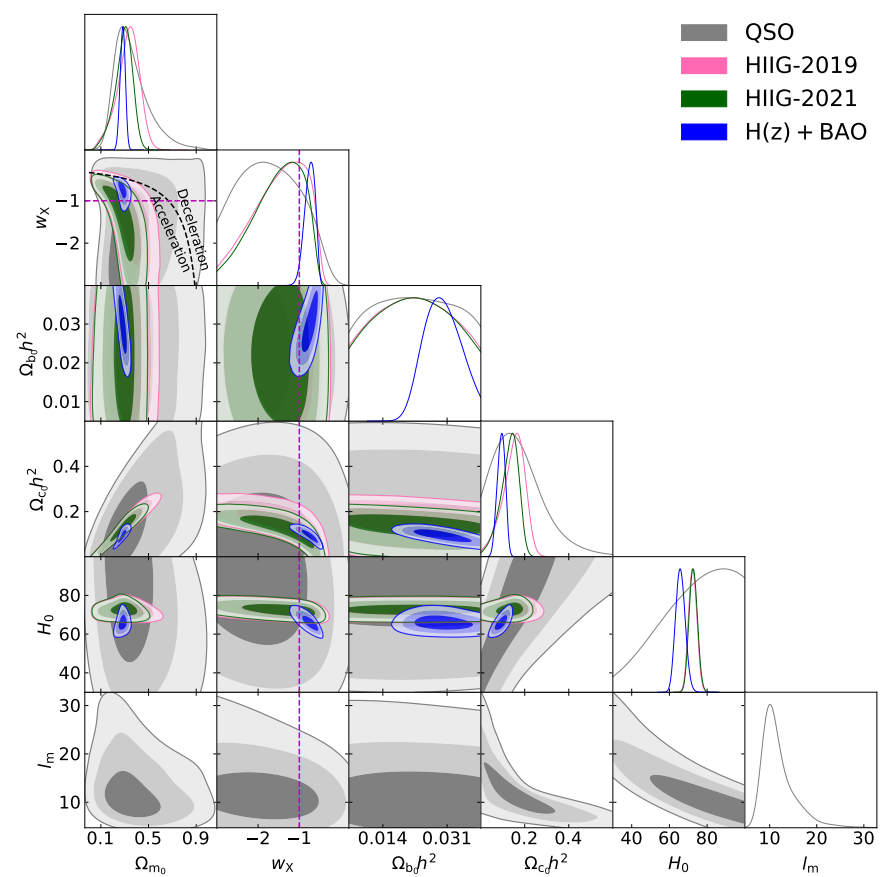

(a)

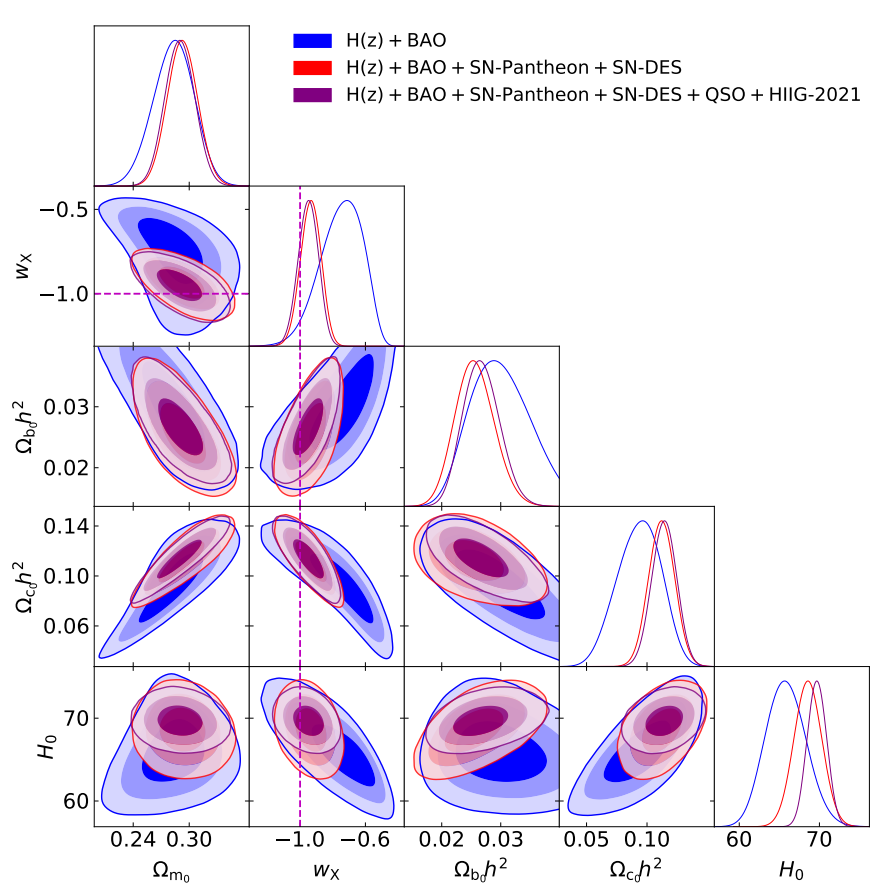

(b)

Figure 3. One-dimensional likelihoods and $1 \sigma, 2 \sigma$, and $3 \sigma$ two-dimensional likelihood confidence contours for flat XCDM. The black dashed line in the left panel is the zero-acceleration line, which divides the parameter space into regions associated with currentlyaccelerating (below left) and currently-decelerating (above right) cosmological expansion. In the right panel, the joint analyses favor currently-accelerating expansion. The magenta lines represent $w_{\mathrm{X}}=-1$, i.e. the flat $\Lambda \mathrm{CDM}$ model.

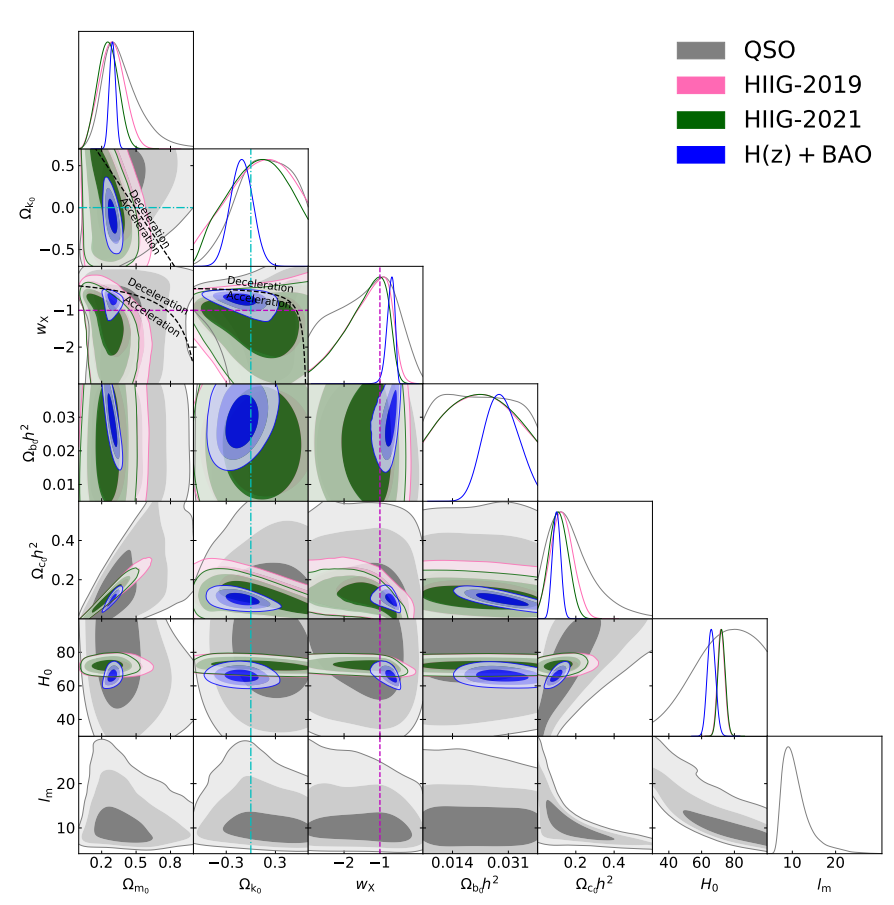

(a)

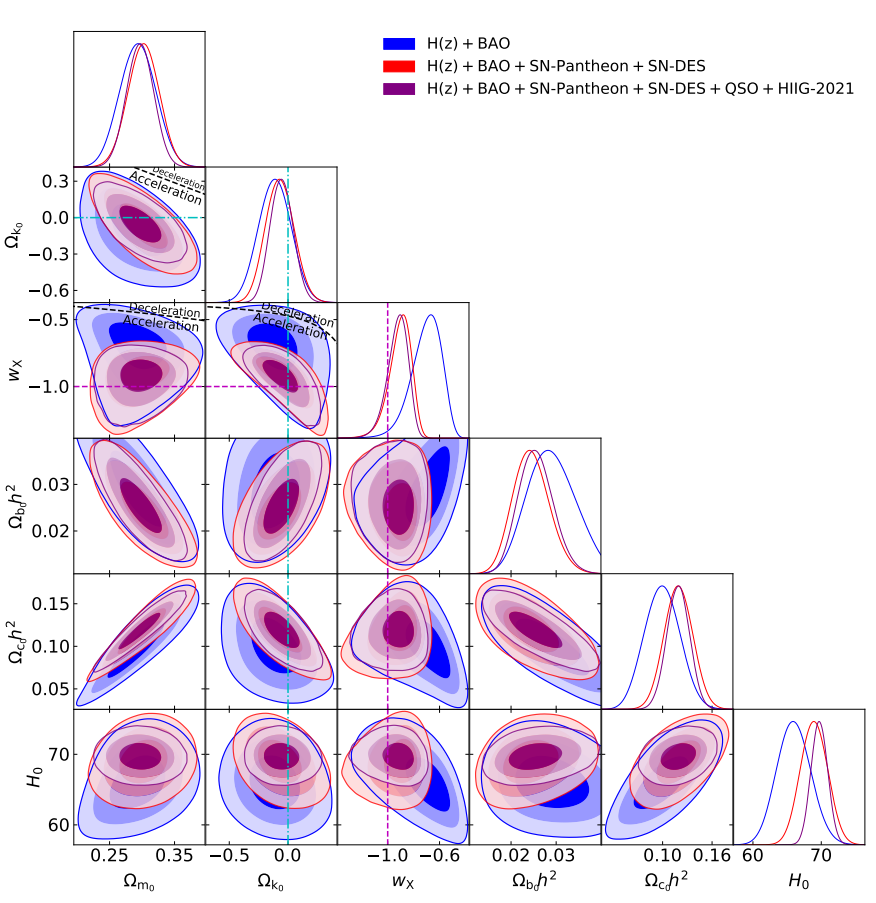

(b)

Figure 4. Same as Fig. 3 but for non-flat XCDM, where the black dashed zero-acceleration lines are computed for the third cosmological parameter set to the $H(z)+$ BAO data best-fitting values listed in Table 1, with currently-accelerating cosmological expansion residing below left. The flat XCDM case is denoted as the cyan dash-dot lines, with closed spatial hypersurfaces either below or to the left. The magenta lines represent $w_{\mathrm{X}}=-1$, i.e. the non-flat $\Lambda \mathrm{CDM}$ model. In all cases except for the QSO only case, almost all of the favored parameter space is associated with currently-accelerating cosmological expansion. 


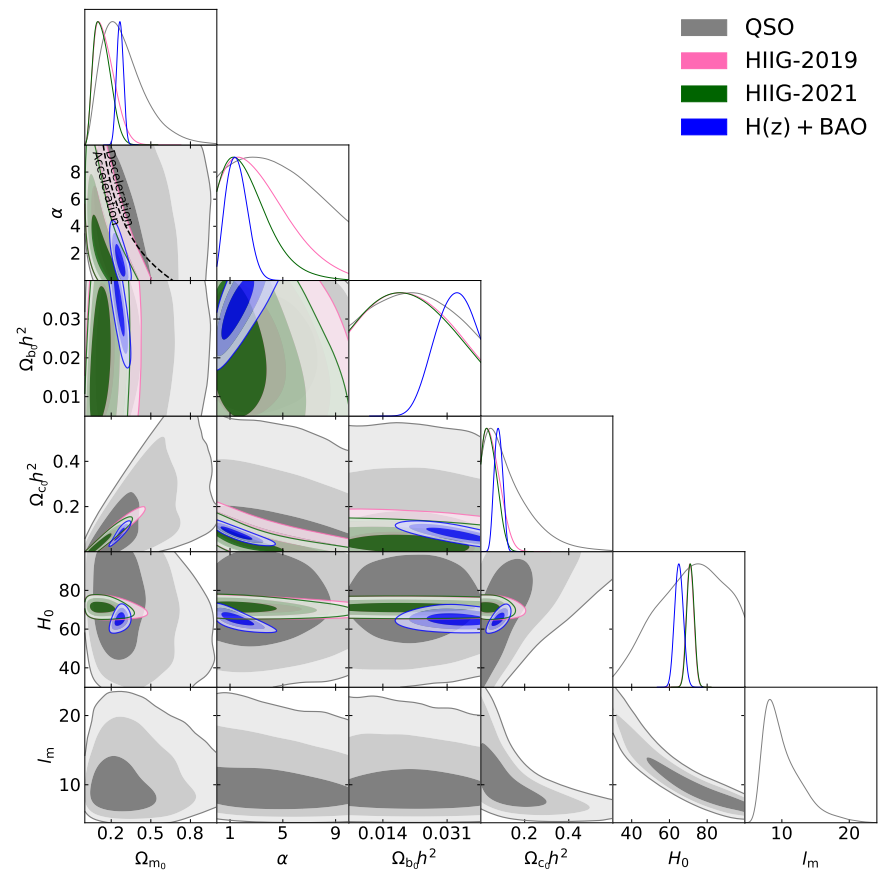

(a)

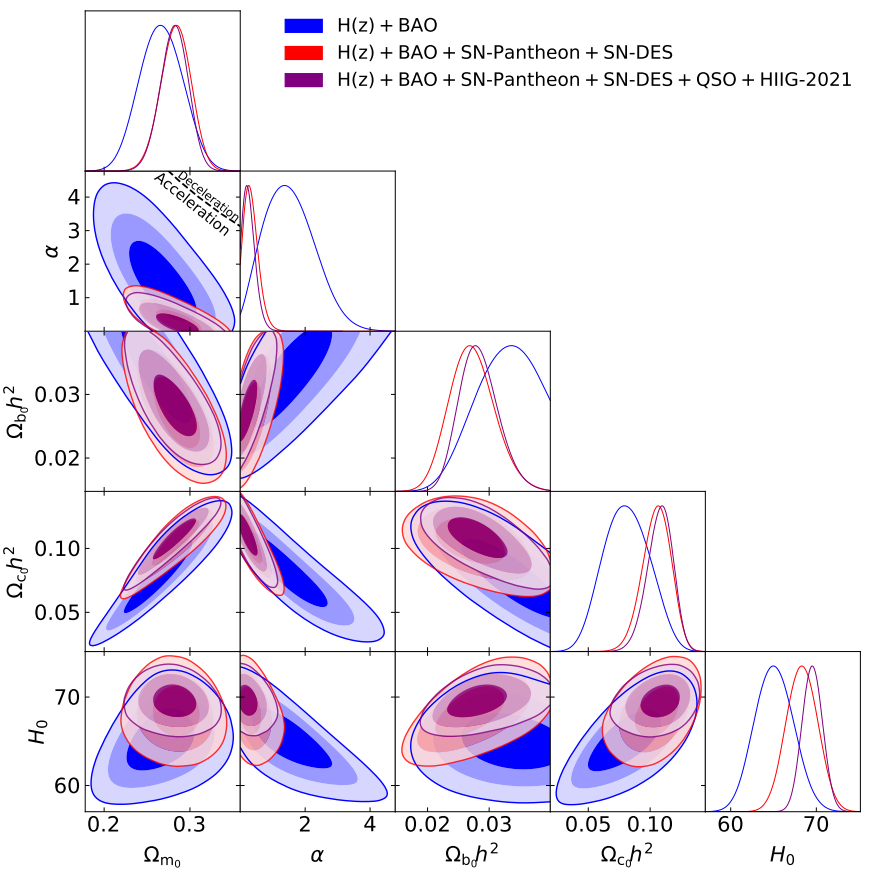

(b)

Figure 5. One-dimensional likelihoods and $1 \sigma, 2 \sigma$, and $3 \sigma$ two-dimensional likelihood confidence contours for flat $\phi$ CDM. The black dashed lines are the zero-acceleration lines, which divides the parameter space into regions associated with currently-accelerating (below left) and currently-decelerating (above right) cosmological expansion. The $\alpha=0$ axis is the flat $\Lambda$ CDM model. In all cases except for the QSO only case, almost all of the favored parameter space is associated with currently-accelerating cosmological expansion.

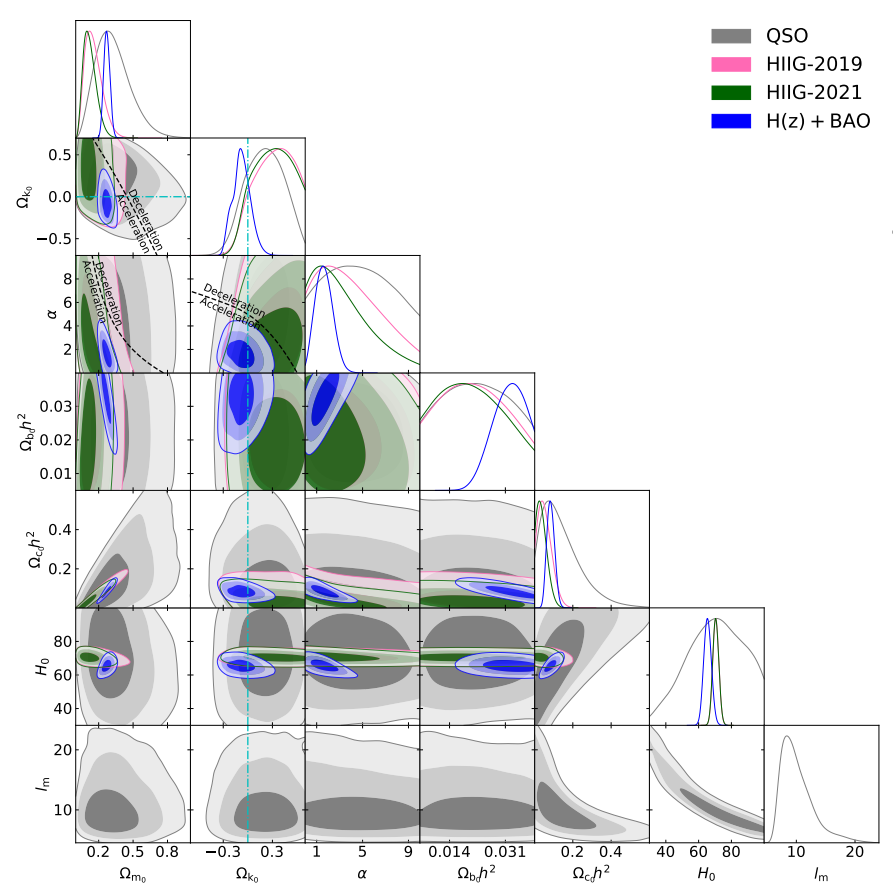

(a)

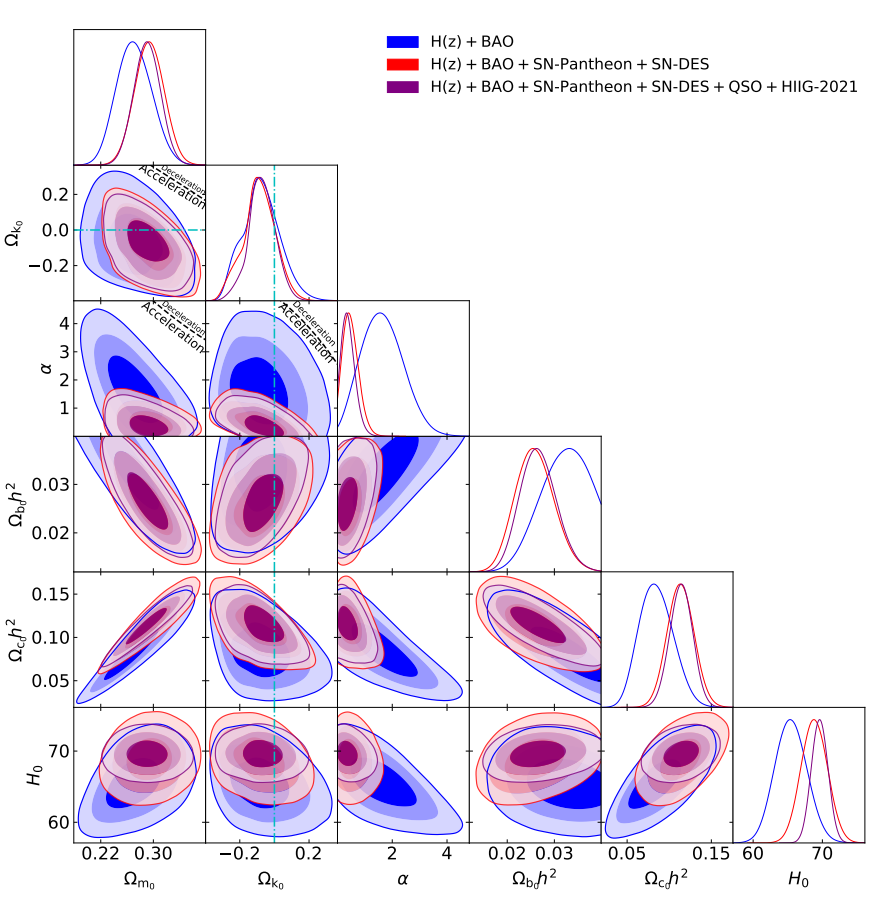

(b)

Figure 6. Same as Fig. 5 but for non-flat $\phi \mathrm{CDM}$, where the black dashed zero-acceleration lines are computed for the third cosmological parameter set to the $H(z)+$ BAO data best-fitting values listed in Table 1. Currently-accelerating cosmological expansion occurs below left of these lines. The cyan dash-dot lines represent the flat $\phi \mathrm{CDM}$ case, with closed spatial geometry either below or to the left. The $\alpha=0$ axis is the non-flat $\Lambda \mathrm{CDM}$ model. In the right panel, the joint analyses favor currently-accelerating expansion. 
We find that the QSO characteristic linear size $l_{\mathrm{m}}$ is relatively model-independent, so QSOs can be treated as approximate standard rulers but the uncertainty in $l_{\mathrm{m}}$ must be accounted for in the analysis.

We also jointly analyzed a total of 1411 measurements, consisting of $31 \mathrm{H}(z), 11 \mathrm{BAO}, 1048 \mathrm{SN}-P a n t h e o n, 20 \mathrm{SN}-$ DES, 120 QSO, and $181 \mathrm{H}$ IIG-2021 data points to constrain cosmological and nuisance parameters in six flat and non-flat cosmological models. We can describe the relatively modelindependent summary features of the constraints obtained from this $H(z)+\mathrm{BAO}+\mathrm{SN}-\mathrm{Pantheon}+\mathrm{SN}-\mathrm{DES}+\mathrm{QSO}+$ H IIG-2021 (HzBSNPDQH) data combination as follows. ${ }^{15}$ First, the constraint on $l_{\mathrm{m}}$ is $l_{\mathrm{m}}=10.93 \pm 0.25 \mathrm{pc}$, which is consistent with the $l_{\mathrm{m}}=11.03 \pm 0.25 \mathrm{pc}$ of Cao et al. (2017). Second, the constraint on $\Omega_{\mathrm{m}_{0}}$ is $\Omega_{\mathrm{m}_{0}}=0.293 \pm 0.021$, which is in good agreement with many other recent measurements (e.g. $0.315 \pm 0.007$ from TT,TE, $\mathrm{TE}+$ lowE + lensing $\mathrm{CMB}$ anisotropy data in the flat $\Lambda \mathrm{CDM}$ model of Planck Collaboration 2020). Third, the determination of $H_{0}$ is $H_{0}=69.7 \pm 1.2 \mathrm{~km} \mathrm{~s}^{-1} \mathrm{Mpc}^{-1}$, which is in better agreement with the estimate of Chen \& Ratra (2011) than with the measurements of Planck Collaboration (2020) and Riess et al. (2021). There is some room for dark energy dynamics or a little spatial curvature energy density, but overall the flat $\Lambda$ CDM model is the best candidate model.

\section{ACKNOWLEDGEMENTS}

We thank Javier de Cruz Pérez, Ana Luisa González-Morán, Narayan Khadka, and Chan-Gyung Park for useful discussions. This work was partially funded by Department of Energy grant DE-SC0011840. The computing for this project was performed on the Beocat Research Cluster at Kansas State University, which is funded in part by NSF grants CNS-1006860, EPS-1006860, EPS-0919443, ACI-1440548, CHE-1726332, and NIH P20GM113109.

\section{DATA AVAILABILITY}

The HiIG data used in this article were provided to us by the authors of González-Morán et al. (2019, 2021). These data will be shared on request to the corresponding author with the permission of the authors of González-Morán et al. $(2019,2021)$.

\section{REFERENCES}

Alam S., et al., 2017, MNRAS, 470, 2617

Amati L., Guidorzi C., Frontera F., Della Valle M., Finelli F., Landi R., Montanari E., 2008, MNRAS, 391, 577

15 The following summary values are obtained with the same method used in Cao et al. (2021b), where we take the summary central value to be the mean of the two of six central-most values. As for the uncertainty, we call the difference between the two central-most values twice the systematic uncertainty and the average of the two central-most error bars the statistical uncertainty, and compute the summary error bar as the quadrature sum of the two uncertainties.
Amati L., D'Agostino R., Luongo O., Muccino M., Tantalo M., 2019, MNRAS, 486, L46

Arjona R., Nesseris S., 2021, Phys. Rev. D, 103, 103539

Ata M., et al., 2018, MNRAS, 473, 4773

Audren B., Lesgourgues J., Benabed K., Prunet S., 2013, J. Cosmology Astropart. Phys., 2013, 001

Avsajanishvili O., Samushia L., Arkhipova N. A., Kahniashvili T., 2015, preprint, (arXiv:1511.09317)

Birrer S., et al., 2020, A\&A, 643, A165

Blas D., Lesgourgues J., Tram T., 2011, J. Cosmology Astropart. Phys., 2011, 034

Blum K., Castorina E., Simonović M., 2020, ApJ, 892, L27

Boruah S. S., Hudson M. J., Lavaux G., 2021, MNRAS,

Breuval L., et al., 2020, A\&A, 643, A115

Calabrese E., Archidiacono M., Melchiorri A., Ratra B., 2012, Phys. Rev. D, 86, 043520

Cao S., Zheng X., Biesiada M., Qi J., Chen Y., Zhu Z.-H., 2017, A\&A, 606, A15

Cao S., Ryan J., Ratra B., 2020, MNRAS, 497, 3191

Cao S., Ryan J., Khadka N., Ratra B., 2021a, MNRAS, 501, 1520

Cao S., Ryan J., Ratra B., 2021b, MNRAS, 504, 300

Carter P., Beutler F., Percival W. J., Blake C., Koda J., Ross A. J., 2018, MNRAS, 481, 2371

Chávez R., Terlevich R., Terlevich E., Bresolin F., Melnick J., Plionis M., Basilakos S., 2014, MNRAS, 442, 3565

Chávez R., Plionis M., Basilakos S., Terlevich R., Terlevich E., Melnick J., Bresolin F., González-Morán A. L., 2016, MNRAS, 462, 2431

Chen G., Ratra B., 2003, ApJ, 582, 586

Chen G., Ratra B., 2011, PASP, 123, 1127

Chen Y., Ratra B., Biesiada M., Li S., Zhu Z.-H., 2016, ApJ, 829, 61

Chen Y., Kumar S., Ratra B., 2017, ApJ, 835, 86

Cuceu A., Farr J., Lemos P., Font-Ribera A., 2019, J. Cosmology Astropart. Phys., 2019, 044

Czerny B., et al., 2021, Acta Physica Polonica A, 139, 389

DES Collaboration 2018, MNRAS, 480, 3879

DES Collaboration 2019a, Phys. Rev. D, 99, 123505

DES Collaboration 2019b, MNRAS, 483, 4866

DES Collaboration 2019c, ApJ, 874, 150

Demianski M., Piedipalumbo E., Sawant D., Amati L., 2021, MNRAS, 506, 903

Denzel P., Coles J. P., Saha P., Williams L. L. R., 2021, MNRAS, 501,784

Dhawan S., Jha S. W., Leibundgut B., 2018, A\&A, 609, A72

Dhawan S., Alsing J., Vagnozzi S., 2021, MNRAS, 506, L1

Di Valentino E., et al., 2021a, Classical and Quantum Gravity, 38,153001

Di Valentino E., Melchiorri A., Silk J., 2021b, ApJ, 908, L9

Domínguez A., et al., 2019, ApJ, 885, 137

du Mas des Bourboux H., et al., 2020, ApJ, 901, 153

eBOSS Collaboration 2021, Phys. Rev. D, 103, 083533

Efstathiou G., 2020, preprint, (arXiv:2007.10716)

Efstathiou G., Gratton S., 2020, MNRAS, 496, L91

Fana Dirirsa F., et al., 2019, ApJ, 887, 13

Farooq O., Mania D., Ratra B., 2015, Ap\&SS, 357, 11

Farooq O., Ranjeet Madiyar F., Crandall S., Ratra B., 2017, ApJ, 835,26

Fernández Arenas D., et al., 2018, MNRAS, 474, 1250

Freedman W. L., 2021, ApJ, 919, 16

Gómez-Valent A., Amendola L., 2018, J. Cosmology Astropart. Phys., 4, 051

González-Morán A. L., et al., 2019, MNRAS, 487, 4669

González-Morán A. L., et al., 2021, MNRAS, 505, 1441

Gordon K. D., Clayton G. C., Misselt K. A., Landolt A. U., Wolff M. J., 2003, ApJ, 594, 279

Gott III J. R., Vogeley M. S., Podariu S., Ratra B., 2001, ApJ, 549,1 
Gurvits L. I., Kellermann K. I., Frey S., 1999, A\&A, 342, 378

Handley W., 2019, Phys. Rev. D, 100, 123517

Harvey D., 2020, MNRAS, 498, 2871

Hogg D. W., 1999, preprint, (arXiv:astro-ph/9905116)

Hu J. P., Wang F. Y., Dai Z. G., 2021, MNRAS, 507, 730

Johnson J. P., Sangwan A., Shankaranarayanan S., 2021, preprint, (arXiv:2102.12367)

Kass R. E., Raftery A. E., 1995, J. Am. Stat. Assoc., 90, 773

Khadka N., Ratra B., 2020a, MNRAS, 492, 4456

Khadka N., Ratra B., 2020b, MNRAS, 497, 263

Khadka N., Ratra B., 2020c, MNRAS, 499, 391

Khadka N., Ratra B., 2021a, preprint, (arXiv:2107.07600)

Khadka N., Ratra B., 2021b, MNRAS, 502, 6140

Khadka N., Yu Z., Zajaček M., Martinez-Aldama M. L., Czerny B., Ratra B., 2021a, MNRAS, 508, 4722

Khadka N., Luongo O., Muccino M., Ratra B., 2021b, J. Cosmology Astropart. Phys., 2021, 042

Khetan N., et al., 2021, A\&A, 647, A72

KiDS Collaboration 2021, A\&A, 649, A88

Kim Y. J., Kang J., Lee M. G., Jang I. S., 2020, ApJ, 905, 104

Lewis A., 2019, preprint, (arXiv:1910.13970)

Li E.-K., Du M., Xu L., 2020, MNRAS, 491, 4960

Li X., Keeley R. E., Shafieloo A., Zheng X., Cao S., Biesiada M., Zhu Z.-H., 2021, MNRAS, 507, 919

Lian Y., Cao S., Biesiada M., Chen Y., Zhang Y., Guo W., 2021, MNRAS, 505, 2111

Lima J. A. S., Alcaniz J. S., 2002, ApJ, 566, 15

Lin W., Ishak M., 2021, J. Cosmology Astropart. Phys., 2021, 009

Lusso E., et al., 2020, A\&A, 642, A150

Lyu M.-Z., Haridasu B. S., Viel M., Xia J.-Q., 2020, ApJ, 900, 160

Mania D., Ratra B., 2012, Physics Letters B, 715, 9

Martínez-Aldama M. L., Czerny B., Kawka D., Karas V., Panda S., Zajaček M., Życki P. T., 2019, ApJ, 883, 170

Mehrabi A., et al., 2022, MNRAS, 509, 224

Melnick J., Terlevich R., Terlevich E., 2000, MNRAS, 311, 629

Moresco M., 2015, MNRAS, 450, L16

Moresco M., et al., 2012, J. Cosmology Astropart. Phys., 8, 006

Moresco M., et al., 2016, J. Cosmology Astropart. Phys., 5, 014

Ooba J., Ratra B., Sugiyama N., 2018a, ApJ, 864, 80

Ooba J., Ratra B., Sugiyama N., 2018b, ApJ, 866, 68

Ooba J., Ratra B., Sugiyama N., 2018c, ApJ, 869, 34

Ooba J., Ratra B., Sugiyama N., 2019, Ap\&SS, 364, 176

Park C.-G., Ratra B., 2018, ApJ, 868, 83

Park C.-G., Ratra B., 2019a, Ap\&SS, 364, 82

Park C.-G., Ratra B., 2019b, Ap\&SS, 364, 134

Park C.-G., Ratra B., 2019c, ApJ, 882, 158

Park C.-G., Ratra B., 2020, Phys. Rev. D, 101, 083508

Pavlov A., Westmoreland S., Saaidi K., Ratra B., 2013, Phys. Rev. D, 88, 123513

Peebles P. J. E., 1984, ApJ, 284, 439

Peebles P. J. E., Ratra B., 1988, ApJ, 325, L17

Perivolaropoulos L., Skara F., 2021, preprint, (arXiv:2105.05208)

Philcox O. H. E., Ivanov M. M., Simonović M., Zaldarriaga M., 2020, J. Cosmology Astropart. Phys., 2020, 032

Planck Collaboration 2020, A\&A, 641, A6

Plionis M., Terlevich R., Basilakos S., Bresolin F., Terlevich E., Melnick J., Chavez R., 2011, MNRAS, 416, 2981

Pogosian L., Zhao G.-B., Jedamzik K., 2020, ApJ, 904, L17

Rameez M., Sarkar S., 2021, Classical and Quantum Gravity, 38, 154005

Rana A., Jain D., Mahajan S., Mukherjee A., 2017, J. Cosmology Astropart. Phys., 2017, 028

Ratra B., Peebles P. J. E., 1988, Phys. Rev. D, 37, 3406

Ratsimbazafy A. L., Loubser S. I., Crawford S. M., Cress C. M., Bassett B. A., Nichol R. C., Väisänen P., 2017, MNRAS, 467, 3239
Riess A. G., Casertano S., Yuan W., Bowers J. B., Macri L., Zinn J. C., Scolnic D., 2021, ApJ, 908, L6

Rigault M., et al., 2015, ApJ, 802, 20

Risaliti G., Lusso E., 2015, ApJ, 815, 33

Risaliti G., Lusso E., 2019, Nature Astronomy, 3, 272

Ryan J., 2021, J. Cosmology Astropart. Phys., 2021, 051

Ryan J., Doshi S., Ratra B., 2018, MNRAS, 480, 759

Ryan J., Chen Y., Ratra B., 2019, MNRAS, 488, 3844

Samushia L., Ratra B., 2010, ApJ, 714, 1347

Sangwan A., Tripathi A., Jassal H. K., 2018, preprint, (arXiv: 1804.09350)

Schöneberg N., Lesgourgues J., Hooper D. C., 2019, J. Cosmology Astropart. Phys., 2019, 029

Scolnic D. M., et al., 2018, ApJ, 859, 101

Seikel M., Clarkson C., Smith M., 2012, J. Cosmol. Astropart. Phys., 2012, 036

Siegel E. R., Guzmán R., Gallego J. P., Orduña López M., Rodríguez Hidalgo P., 2005, MNRAS, 356, 1117

Simon J., Verde L., Jimenez R., 2005, Phys. Rev. D, 71, 123001

Singh A., Sangwan A., Jassal H. K., 2019, J. Cosmology Astropart. Phys., 2019, 047

Sinha S., Banerjee N., 2021, J. Cosmology Astropart. Phys., 2021, 060

Solà Peracaula J., de Cruz Pérez J., Gómez-Valent A., 2018, MNRAS, 478, 4357

Solà Peracaula J., Gómez-Valent A., de Cruz Pérez J., 2019, Physics of the Dark Universe, 25, 100311

Stern D., Jimenez R., Verde L., Kamionkowski M., Stanford S. A., 2010, J. Cosmology Astropart. Phys., 2, 008

Terlevich R., Terlevich E., Melnick J., Chávez R., Plionis M., Bresolin F., Basilakos S., 2015, MNRAS, 451, 3001

Tsiapi P., et al., 2021, MNRAS, 506, 5039

Ureña-López L. A., Roy N., 2020, Phys. Rev. D, 102, 063510

Vagnozzi S., Di Valentino E., Gariazzo S., Melchiorri A., Mena O., Silk J., 2021a, Physics of the Dark Universe, 33, 100851

Vagnozzi S., Loeb A., Moresco M., 2021b, ApJ, 908, 84

Velasquez-Toribio A. M., Fabris J. C., 2020, European Physical Journal C, 80, 1210

Vishwakarma R. G., 2001, Classical and Quantum Gravity, 18, 1159

Wang J. S., Wang F. Y., Cheng K. S., Dai Z. G., 2016, A\&A, 585, A68

Wang F. Y., Hu J. P., Zhang G. Q., Dai Z. G., 2021, preprint, (arXiv:2106.14155)

Wei J.-J., 2018, ApJ, 868, 29

Yang T., Banerjee A., Ó Colgáin E., 2020, Phys. Rev. D, 102, 123532

Yu H., Ratra B., Wang F.-Y., 2018, ApJ, 856, 3

Yu Z., et al., 2021, MNRAS, 507, 3771

Zajaček M., et al., 2021, ApJ, 912, 10

Zeng H., Yan D., 2019, ApJ, 882, 87

Zhai Z., Blanton M., Slosar A., Tinker J., 2017, ApJ, 850, 183

Zhang X., Huang Q.-G., 2021, Phys. Rev. D, 103, 043513

Zhang C., Zhang H., Yuan S., Liu S., Zhang T.-J., Sun Y.-C., 2014, Research in Astronomy and Astrophysics, 14, 1221

Zhang B. R., Childress M. J., Davis T. M., Karpenka N. V., Lidman C., Schmidt B. P., Smith M., 2017, MNRAS, 471, 2254

Zhu Z.-H., Fujimoto M.-K., 2002, ApJ, 581, 1

This paper has been typeset from a $\mathrm{TEX}_{\mathrm{E}} / \mathrm{LATEX}$ file prepared by the author. 